\title{
PANCHROMATIC OBSERVATIONS OF THE TEXTBOOK GRB 110205A: CONSTRAINING PHYSICAL MECHANISMS OF PROMPT EMISSION AND AFTERGLOW
}

W. Zheng ${ }^{1}$, R. F. Shen ${ }^{2}$, T. Sakamoto ${ }^{3,4,5}$, A. P. Beardmore ${ }^{6}$, M. De Pasquale ${ }^{7}$, X. F. Wu ${ }^{8,9}$, J. Gorosabel $^{10}$, Y. Urata ${ }^{11}$

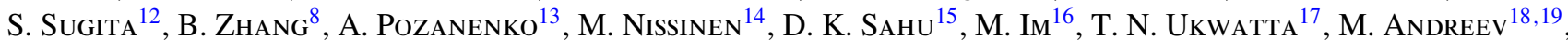

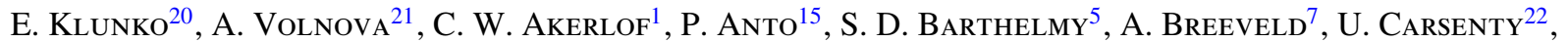

S. Castillo-Carrión ${ }^{23}$, A. J. Castro-Tirado ${ }^{10}$, M. M. Chester ${ }^{24}$, C. J. Chuang ${ }^{11}$, R. Cunniffe ${ }^{10}$, A. De Ugarte Postigo ${ }^{25}$, R. Duffard ${ }^{10}$, H. Flewelling ${ }^{26}$, N. Gehrels ${ }^{5}$, T. Güver ${ }^{27}$, S. GuZiY ${ }^{10}$, V. P. Hentunen ${ }^{14}$, K. Y. HuAnG ${ }^{28}$, M. JelíneK ${ }^{10}$,

T. S. Koch $^{24}$, P. KubáneK ${ }^{10}$, P. Kuin ${ }^{7}$, T. A. McKay ${ }^{1}$, S. Mottola ${ }^{22}$, S. R. OAtes ${ }^{7}$, P. O’Brien ${ }^{6}$, M. Ohno ${ }^{29}$, M. J. Page ${ }^{7}$,

S. B. Pandey ${ }^{30}$, C. Pérez del Pulgar ${ }^{23}$, W. Rujopakarn ${ }^{31}$, E. RykofF ${ }^{32}$, T. Salmi ${ }^{14}$, R. Sánchez-Ramírez ${ }^{10}$,

B. E. Schaefer ${ }^{33}$, A. Sergeev ${ }^{18,19}$, E. Sonbas ${ }^{5,34,35}$, A. Sota ${ }^{10}$, J. C. Tello ${ }^{10}$, K. YamaokA ${ }^{36}$, S. A. Yost ${ }^{37}$, And F. Yuan ${ }^{38,39}$

${ }^{1}$ Department of Physics, University of Michigan, 450 Church Street, Ann Arbor, MI 48109, USA; zwk@ umich.edu

${ }^{2}$ Department of Astronomy and Astrophysics, University of Toronto, Toronto, Ontario M5S 3H4, Canada; rfshen@ astro.utoronto.ca

${ }^{3}$ Center for Research and Exploration in Space Science and Technology (CRESST), NASA Goddard Space Flight Center, Greenbelt, MD 20771, USA

${ }^{4}$ Joint Center for Astrophysics, University of Maryland, Baltimore County, 1000 Hilltop Circle, Baltimore, MD 21250, USA

${ }^{5}$ NASA Goddard Space Flight Center, Greenbelt, MD 20771, USA

${ }^{6}$ Department of Physics and Astronomy, University of Leicester, Leicester LE1 7RH, UK

${ }^{7}$ Mullard Space Science Laboratory, University College London, Holmbury Road, Holmbury St. Mary, Dorking RH5 6NT, UK

${ }^{8}$ Department of Physics and Astronomy, University of Nevada Las Vegas, Las Vegas, NV 89154, USA; zhang@physics.unlv.edu

${ }^{9}$ Purple Mountain Observatory, Chinese Academy of Sciences, Nanjing 210008, China

${ }^{10}$ Instituto de Astrofísica de Andalucía (IAA-CSIC), 18008 Granada, Spain

${ }^{11}$ Institute of Astronomy, National Central University, Chung-Li 32054, Taiwan

${ }^{12}$ EcoTopia Science Institute, Nagoya University, Furo-cho, chikusa, Nagoya 464-8603, Japan

${ }^{13}$ Space Research Institute (IKI), 84/32 Profsoyuznaya St., Moscow 117997, Russia

14 Taurus Hill Observatory, Härkämäentie 88, 79480 Kangaslampi, Finland

${ }^{15}$ CREST, Indian Institute of Astrophysics, Koramangala, Bangalore 560034, India

${ }^{16}$ Center for the Exploration of the Origin of the Universe, Department of Physics and Astronomy, FPRD, Seoul National University, Shillim-dong, San 56-1, Kwanak-gu, Seoul, Republic of Korea

${ }^{17}$ Department of Physics and Astronomy, Michigan State University, East Lansing, MI 48824, USA

${ }^{18}$ Terskol Branch of Institute of Astronomy of RAS, Kabardino-Balkaria Republic 361605, Russian Federation

${ }^{19}$ International Centre of Astronomical and Medico-Ecological Research of NASU, 27 Akademika Zabolotnoho St., 03680 Kyiv, Ukraine

${ }^{20}$ Institute of Solar-Terrestrial Physics, Lermontov St., 126a, Irkutsk 664033, Russia

${ }^{21}$ Sternberg Astronomical Institute, Moscow State University, Universitetsky pr., 13, Moscow 119992, Russia

${ }^{22}$ Institute of Planetary Research, DLR, 12489 Berlin, Germany

${ }^{23}$ Department of EVLT, Universidad de Málaga, Campus de Teatinos, Málaga, Spain

${ }^{24}$ Department of Astronomy and Astrophysics, Penn State University, 525 Davey Laboratory, University Park, PA 16802, USA

${ }^{25}$ Dark Cosmology Centre, Niels Bohr Institute, University of Copenhagen, Juliane Maries Vej 30, DK-2100 Copenhagen O, Denmark

${ }^{26}$ Institute for Astronomy, 2680 Woodlawn Avenue, Honolulu, HI 96822, USA

${ }^{27}$ Department of Astronomy, University of Arizona, 933 North Cherry Avenue, Tucson, AZ 85721, USA

${ }_{28}$ Academia Sinica Institute of Astronomy and Astrophysics, Taipei 106, Taiwan

${ }^{29}$ Japan Aerospace Exploration Agency, Institute of Space and Astronautical Science, 3-1-1 Yoshinodai, Chuo-ku, Sagamihara, Kanagawa 252-5210, Japan

${ }^{30}$ Aryabhatta Research Institute of Observational Sciences (ARIES), Manora Peak, Nainital 263129, India

${ }^{31}$ Steward Observatory, The University of Arizona, Tucson, AZ 85721, USA

${ }^{32}$ E. O. Lawrence Berkeley National Laboratoy, 1 Cyclotron Road, Berkeley, CA 94720, USA

${ }^{33}$ Department of Physics and Astronomy, Louisiana State University, Baton Rouge, LA 70803, USA

${ }^{34}$ Department of Physics, University of Adiyaman, 02040 Adiyaman, Turkey

${ }^{35}$ Universities Space Research Association, 10211 Wincopin Circle, Suite 500, Columbia, MD 21044-3432, USA

${ }^{36}$ Department of Physics and Mathematics, Aoyama Gakuin University, 5-10-1 Fuchinobe, Chuo-ku, Sagamihara, Kanagawa 252-5258, Japan

${ }^{37}$ Department of Physics, College of St. Benedict, Collegeville, MN 56321, USA

${ }^{38}$ Research School of Astronomy and Astrophysics, The Australian National University, Weston Creek, ACT 2611, Australia

${ }^{39}$ ARC Centre of Excellence for All-sky Astrophysics (CAASTRO)

Received 2011 November 1; accepted 2012 March 22; published 2012 May 9

\section{ABSTRACT}

We present a comprehensive analysis of a bright, long-duration $\left(T_{90} \sim 257 \mathrm{~s}\right)$ GRB $110205 \mathrm{~A}$ at redshift $z=2.22$. The optical prompt emission was detected by Swift/UVOT, ROTSE-IIIb, and BOOTES telescopes when the gamma-ray burst (GRB) was still radiating in the $\gamma$-ray band, with optical light curve showing correlation with $\gamma$-ray data. Nearly $200 \mathrm{~s}$ of observations were obtained simultaneously from optical, $\mathrm{X}$-ray, to $\gamma$-ray $(1 \mathrm{eV}$ to $5 \mathrm{MeV}$ ), which makes it one of the exceptional cases to study the broadband spectral energy distribution during the prompt emission phase. In particular, we clearly identify, for the first time, an interesting two-break energy spectrum, roughly consistent with the standard synchrotron emission model in the fast cooling regime. Shortly after prompt emission $(\sim 1100 \mathrm{~s})$, a bright $(R=14.0)$ optical emission hump with very steep rise $(\alpha \sim 5.5)$ was observed, which we interpret as the reverse shock (RS) emission. It is the first time that the rising phase of an RS component has been closely observed. The full optical and X-ray afterglow light curves can be interpreted within the standard reverse shock (RS) + forward shock (FS) model. In general, the high-quality prompt and afterglow data allow us to apply the standard fireball model to extract valuable information, including the radiation mechanism (synchrotron), 
radius of prompt emission $\left(R_{\mathrm{GRB}} \sim 3 \times 10^{13} \mathrm{~cm}\right)$, initial Lorentz factor of the outflow $\left(\Gamma_{0} \sim 250\right)$, the composition of the ejecta (mildly magnetized), the collimation angle, and the total energy budget.

Key word: gamma-ray burst: individual (GRB 110205A)

Online-only material: color figures

\section{INTRODUCTION}

Gamma-ray bursts (GRBs) are extremely luminous explosions in the universe. A standard fireball model (e.g., Rees \& Mészáros 1992, 1994; Mészáros \& Rees 1997; Wijers et al. 1997; Sari et al. 1998; see, e.g., Zhang \& Mészáros 2004; Mészáros 2006 for reviews) has been developed following their discovery in 1973 (Klebesadel et al. 1973) to explain their observational nature. Generally, the prompt emission can be modeled as originating from internal shocks or the photosphere of the fireball ejecta or magnetic dissipation from a magnetically dominated jet, while the afterglow emission originates from external shocks that may include both forward shock (FS) and reverse shock (RS) components (Mészáros \& Rees 1997, 1999; Sari \& Piran 1999).

The leading radiation mechanisms of the GRB prompt emission are synchrotron radiation, synchrotron self-Compton (SSC), and Compton upscattering of a thermal seed photon source (e.g., Zhang 2011 for a review). All these mechanisms give a "non-thermal" nature to the GRB prompt spectrum. Observationally, the prompt spectrum in the $\gamma$-ray band can be fit with a smoothly broken power law called the Band function (Band et al. 1993). Since this function is characterized by a single break energy, it cannot adequately fit the spectrum if the spectral distribution is too complex. For example, the synchrotron mechanism predicts an overall power-law spectrum characterized by several break frequencies: $v_{a}$ (self-absorption frequency), $v_{m}$ (the frequency of minimum electron injection energy), and $v_{c}$ (cooling frequency) (Sari \& Esin 2001). However, due to instrumental and observational constraints, it is almost impossible to cover the entire energy range and re-construct the prompt spectrum with all three predicted break points. Thus, despite its limited number of degrees of freedom, the Band function is an empirically good description for most GRBs.

The Swift mission (Gehrels et al. 2004), thanks to its rapid and precise localization capability, performs simultaneous observations in the optical to $\gamma$-ray bands, allowing broadband observations of the prompt phase much more frequently than previous GRB probes. This energy range may also span up to six orders of magnitude (e.g., GRB 090510, Abdo et al. 2009; De Pasquale et al. 2010) if a GRB is observed by both the Swift and Fermi satellites (Atwood et al. 2009; Meegan et al. 2009). Some prompt observations have shown signatures of a synchrotron spectrum from the break energies $\left(v_{m}, v_{c}\right)$ (e.g., GRB 080928, Rossi et al. 2011). Prompt optical observations can also be used to constrain the self-absorption frequency $v_{a}$ (Shen \& Zhang 2009), but, so far, no GRB has been observed clearly with more than two break energies in the prompt spectrum. Meanwhile, early-time observations in the optical band provide a greater chance to detect RS emission, which has only been observed for a few bursts since the first detection in GRB 990123 (Akerlof et al. 1999).

Here we report on the analysis of the long-duration GRB 110205A triggered by the Swift/BAT (Barthelmy et al. 2005). Both prompt and afterglow emissions are detected with good data sampling. Broadband energy coverage over six orders of magnitude ( $1 \mathrm{eV}$ to $5 \mathrm{MeV}$ ) during prompt emission makes this GRB a rare case from which we can study the prompt spectrum in great detail. Its bright optical $(R=14.0 \mathrm{mag})$ and X-ray afterglows allow us to test the different external shock models and to constrain the physical parameters of the fireball model.

Throughout this paper, we adopt a standard cosmology model with $H_{0}=71 \mathrm{~km} \mathrm{~s}^{-1} \mathrm{Mpc}^{-1}, \Omega_{M}=0.27$, and $\Omega_{\Lambda}=0.73$. We use the usual power-law representation of flux density $F(v) \propto t^{\alpha} v^{-\beta}$ for the further analysis. All errors are given at the $1 \sigma$ confidence level unless otherwise stated.

\section{OBSERVATIONS AND DATA REDUCTIONS}

\subsection{Observations}

At 02:02:41 UT on 2011 February $5\left(T_{0}\right)$, the Swift/BAT triggered and located GRB 110205A (trigger $=444643$; Beardmore et al. 2011). The BAT light curve shows many overlapping peaks with a general slow rise starting at $T_{0}-120 \mathrm{~s}$, with the highest peak at $T_{0}+210 \mathrm{~s}$, and ending at $T_{0}+1500 \mathrm{~s}$. $T_{90}$ $(15-350 \mathrm{keV})$ is $257 \pm 25 \mathrm{~s}$ (estimated error including systematics). GRB 110205A was also detected by WAM (Sugita et al. 2011, also included in our analysis) on board Suzaku (Yamaoka et al. 2009) and Konus-Wind (Golenetskii et al. 2011; Pal'shin 2011 ) in the $\gamma$-ray band. A bright, uncataloged X-ray afterglow was promptly identified by the X-Ray Telescope (Burrows et al. 2005a) $155.4 \mathrm{~s}$ after the burst (Beardmore et al. 2011). The UVOT (Roming et al. 2005) revealed an optical afterglow $164 \mathrm{~s}$ after the burst at location R.A. (J2000) $=10^{\mathrm{h}} 58^{\mathrm{m}} 31^{\mathrm{s}} .12$, decl. $(\mathrm{J} 2000)=+67^{\circ} 31^{\prime} 31^{\prime \prime} .2$ with a $90 \%$ confidence error radius of about 0.63 arcsec (Beardmore et al. 2011), which was later seen to re-brighten (Chester \& Beardmore 2011).

ROTSE-IIIb, located at the McDonald Observatory, Texas, responded to GRB $110205 \mathrm{~A}$ promptly and confirmed the optical afterglow (Schaefer et al. 2011). The first image started at 02:04:03.4 UT, 82.0 s after the burst (8.4 s after the GCN notice time). The optical afterglow was observed to re-brighten dramatically to $14.0 \mathrm{mag} \sim 1100 \mathrm{~s}$ after the burst, as was also reported by other groups (e.g., Klotz et al. 2011a, 2011b; Andreev et al. 2011). ROTSE-IIIb continued monitoring the afterglow until it was no longer detectable, $1.5 \mathrm{hr}$ after the trigger.

Ground-based optical follow-up observations were also performed by different groups with various instruments, some of which are presented by Cucchiara et al. (2011) and Gendre et al. (2011). In this paper, the optical data include Global Rent-a-Scope 005 telescope at New Mexico (Hentunen et al. 2011); $1 \mathrm{~m}$ telescope at Mt. Lemmon Optical Astronomy Observatory (Im \& Urata 2011; Lee et al. 2010); Lulin One-meter Telescope (LOT; Huang et al. 2005; Urata et al. 2011); $0.61 \mathrm{~m}$ Lightbuckets rental telescope LB0001 in Rodeo, NM (Ukwatta et al. 2011); 2 m Himalayan Chandra Telescope (HCT; Sahu \& Anto 2011); Zeiss-600 telescope at Mt. Terskol observatory (Andreev et al. 2011); and $1.6 \mathrm{~m}$ AZT-33IK telescope at Sayan Solar observatory, Mondy 


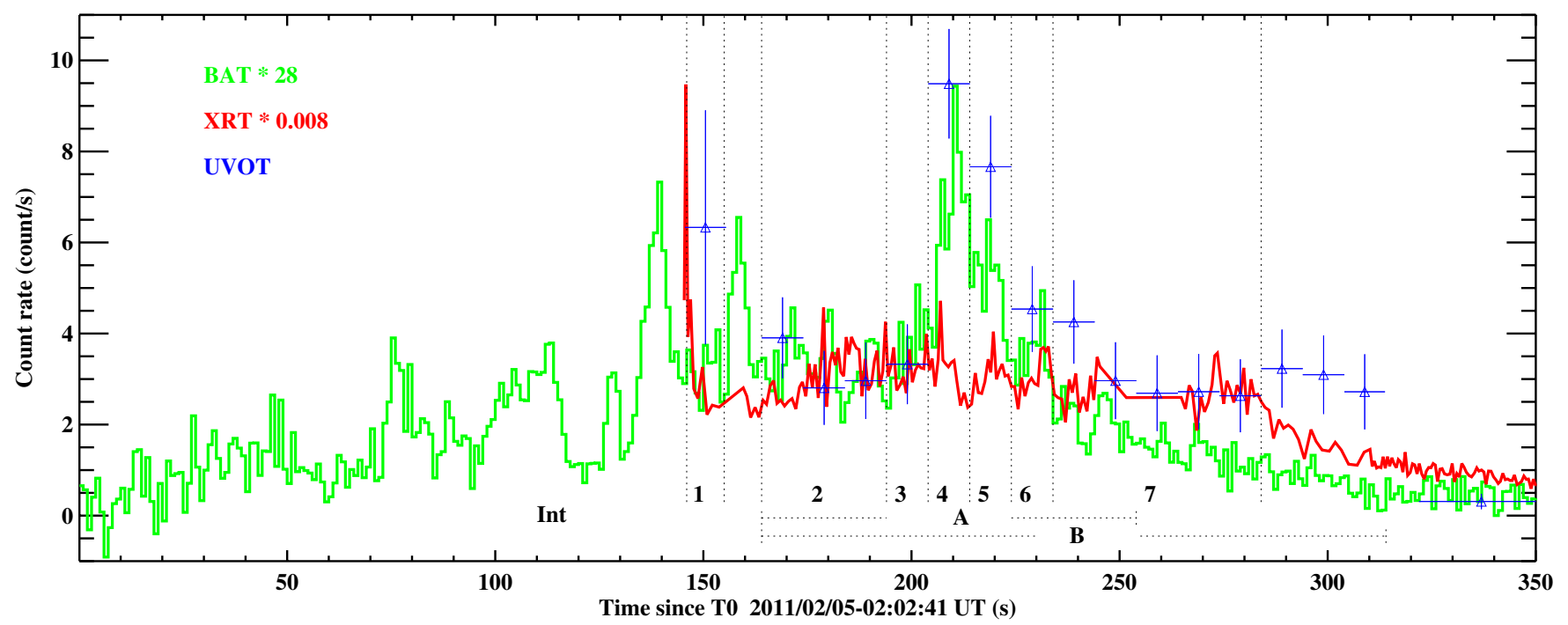

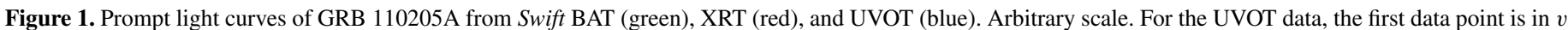

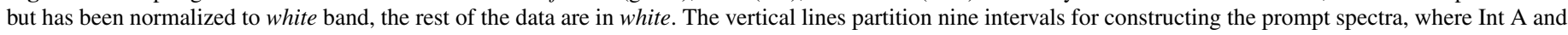
$\mathrm{B}$ are averaged ones.

(A color version of this figure is available in the online journal.)

(Volnova et al. 2011), as well as Burst Observer and Optical Transient Exploring System (BOOTES 1 and 2 telescopes), $1.23 \mathrm{~m}$ and $2.2 \mathrm{~m}$ telescope at Calar Alto Observatory, and $1.5 \mathrm{~m}$ OSN telescope, which are not reported in the GCNs.

The redshift measurement of GRB 110205A was reported by three independent groups with $z=1.98$ (Silva et al. 2011), $z=$ 2.22 (Cenko et al. 2011; Cucchiara et al. 2011), and $z=2.22$ (Vreeswijk et al. 2011). Here we adopt $z=2.22$, for which two observations are in very close agreement.

\subsection{Data Reductions}

The data from Swift and Suzaku, including UVOT, XRT, BAT, and WAM (50 keV to $5 \mathrm{MeV}$ ), were processed with the standard HEAsoft software (version 6.10). The BAT and XRT data were further automatically processed by the Burst Analyser pipeline $^{40}$ (Evans et al. 2007, 2009, 2010), with the light curves background subtracted. For the XRT data, Windowed Timing (WT) data and Photon Counting (PC) data were processed separately. Pile-up corrections were applied if necessary, especially at early times when the source was very bright. The UVOT data were also processed with the standard procedures. A count rate was extracted within a radius of 3 or 5 arcsec depending on the source brightness around the best UVOT coordinates. The data in each filter were binned with $\delta \mathrm{t} / \mathrm{t}=$ 0.2 and then converted to flux density using a UVOT GRB spectral model (Poole et al. 2008; Breeveld et al. 2010, 2011). The UVOT photometry result is listed in Table 1.

For the ground-based optical data, different methods were used for each instrument. For the ROTSE data, the raw images were processed using the standard ROTSE software pipeline. Image co-adding was performed if necessary to obtain a reasonable signal-to-noise ratio. Photometry was then extracted using the method described in Quimby et al. (2006). Other optical data were processed using the standard procedures provided by the IRAF ${ }^{41}$ software. A differential aperture photometry was

\footnotetext{
40 http://www.swift.ac.uk/burst analyser/

41 IRAF is distributed by the National Optical Astronomy Observatory, which is operated by the Association of Universities for Research in Astronomy, Inc., under cooperative agreement with the National Science Foundation.
}

applied with the DAOPHOT package in IRAF. Reference stars were calibrated using the photometry data from Sloan Digital Sky Survey (Smith et al. 2002). Clear $(C)$ band data were calibrated to $R$ band. All ground-based photometry results are listed in Table 2.

The spectral fitting, including WAM, BAT, XRT, and optical data, was performed using Xspec (version 12.5). We constructed a set of prompt emission spectra over nine time intervals during which the optical data were available. Data from each instrument were re-binned to the same time intervals. In the afterglow phase, spectral energy distributions (SEDs) in four different epochs were constructed when we have the best coverage of multi-band data from optical to X-rays: $550 \mathrm{~s}, 1.1 \mathrm{ks}, 5.9 \mathrm{ks}$, and $35 \mathrm{ks}$. All the spectral fittings were carried out under Xspec using $\chi^{2}$ statistics, except the $1.1 \mathrm{ks}$ SED of the afterglow, for which $C$-statistics was used.

\section{MULTI-WAVELENGTH DATA ANALYSIS}

\subsection{Broadband Prompt Emission from Optical to $\gamma$-Rays}

Thanks to its long duration $\left(T_{90}=257 \pm 25 \mathrm{~s}\right), \mathrm{GRB} 110205 \mathrm{~A}$ was also detected by XRT and UVOT during the prompt emission phase starting from $155.4 \mathrm{~s}$ and $164 \mathrm{~s}$ after the trigger, respectively. Both XRT and UVOT obtained nearly $200 \mathrm{~s}$ of high-quality and well-sampled data during the prompt phase. ROTSE-IIIb and BOOTES also detected the optical prompt emission $82.0 \mathrm{~s}$ and $102 \mathrm{~s}$ after the trigger, respectively. Together with the $\gamma$-ray data collected by BAT (15-150 keV) and Suzaku/WAM (50 keV to $5 \mathrm{MeV}$ ), these multi-band prompt emission data cover six orders of magnitude in energy, which allow us to study the temporal and spectral properties of prompt emission in great detail.

Figure 1 shows the prompt light curves from Swift BAT (green), XRT (red), and UVOT (blue). The BAT light curve shows multiple peaks until at least $T_{0}+300 \mathrm{~s}$ with a peak count rate at $T_{0}+210 \mathrm{~s}$. The XRT data show a decay phase from a very bright peak at the start of XRT observations, followed by smaller peaks with complicated variability. The UVOT observations were performed mainly in the white band, 
Table 1

Photomertric Observations for GRB 110205A from Swift/UVOT

\begin{tabular}{|c|c|c|c|c|c|c|c|c|c|}
\hline $\begin{array}{l}T-T_{0} \\
\text { (s) }\end{array}$ & $\begin{array}{l}\text { Error } \\
\text { (s) }\end{array}$ & Count & Error & Filter & $\begin{array}{c}T-T_{0} \\
\quad \text { (s) }\end{array}$ & $\begin{array}{l}\text { Error } \\
\text { (s) }\end{array}$ & Count & Error & Filter \\
\hline 169.0 & 5.0 & 3.905 & 0.890 & white & 24517.0 & 149.9 & 1.133 & 0.130 & white \\
\hline 179.0 & 5.0 & 2.807 & 0.812 & white & 24734.3 & 63.5 & 1.037 & 0.198 & white \\
\hline 189.0 & 5.0 & 2.963 & 0.840 & white & 35675.8 & 149.9 & 0.654 & 0.122 & white \\
\hline 199.0 & 5.0 & 3.326 & 0.877 & white & 35979.3 & 149.9 & 0.653 & 0.123 & white \\
\hline 209.0 & 5.0 & 9.487 & 1.203 & white & 36284.1 & 149.9 & 0.474 & 0.089 & white \\
\hline 219.0 & 5.0 & 7.664 & 1.120 & white & 75564.8 & 149.9 & 0.234 & 0.080 & white \\
\hline 229.0 & 5.0 & 4.538 & 0.939 & white & 75868.3 & 149.9 & 0.256 & 0.081 & white \\
\hline 239.0 & 5.0 & 4.255 & 0.916 & white & 76171.9 & 149.9 & 0.316 & 0.083 & white \\
\hline 249.0 & 5.0 & 2.964 & 0.845 & white & 87890.9 & 92.9 & 0.177 & 0.099 & white \\
\hline 259.0 & 5.0 & 2.689 & 0.831 & white & 99845.1 & 88.9 & 0.085 & 0.100 & white \\
\hline 269.0 & 5.0 & 2.722 & 0.829 & white & 110704.6 & 322.4 & 0.106 & 0.052 & white \\
\hline 279.0 & 5.0 & 2.633 & 0.802 & white & 117007.3 & 147.4 & 0.008 & 0.075 & white \\
\hline 289.0 & 5.0 & 3.229 & 0.858 & white & 122512.3 & 237.9 & 0.028 & 0.059 & white \\
\hline 299.0 & 5.0 & 3.095 & 0.863 & white & 128037.7 & 322.4 & 0.097 & 0.052 & white \\
\hline 308.9 & 4.9 & 2.720 & 0.826 & white & 133882.8 & 299.9 & 0.100 & 0.054 & white \\
\hline 611.8 & 9.9 & 20.129 & 1.184 & white & 139643.2 & 305.9 & 0.034 & 0.052 & white \\
\hline 784.5 & 9.9 & 63.173 & 2.257 & white & 145379.3 & 319.4 & 0.047 & 0.051 & white \\
\hline 950.3 & 74.8 & 88.866 & 1.043 & white & 151192.4 & 308.4 & 0.167 & 0.055 & white \\
\hline 1190.9 & 9.9 & 95.569 & 3.037 & white & 156929.3 & 321.9 & 0.038 & 0.051 & white \\
\hline 1365.9 & 9.9 & 78.130 & 2.615 & white & 162706.6 & 321.4 & 0.041 & 0.051 & white \\
\hline 1538.4 & 9.9 & 64.515 & 2.290 & white & 168487.5 & 320.9 & 0.042 & 0.051 & white \\
\hline 1711.7 & 9.9 & 52.993 & 2.027 & white & 185834.0 & 315.4 & 0.006 & 0.051 & white \\
\hline 5166.4 & 99.9 & 10.865 & 0.283 & white & 203156.9 & 319.9 & 0.088 & 0.052 & white \\
\hline 6602.8 & 99.9 & 7.511 & 0.246 & white & 209526.6 & 120.9 & 0.019 & 0.083 & white \\
\hline 11906.7 & 149.9 & 2.834 & 0.152 & white & 220477.0 & 322.9 & 0.022 & 0.050 & white \\
\hline 12210.4 & 149.9 & 2.752 & 0.150 & white & 232055.5 & 315.9 & 0.042 & 0.052 & white \\
\hline 12513.8 & 149.9 & 2.755 & 0.151 & white & & & & & white \\
\hline 150.5 & 4.9 & 1.107 & 0.449 & $v$ & 52773.8 & 149.9 & 0.077 & 0.039 & $v$ \\
\hline 661.9 & 9.9 & 6.853 & 0.643 & $v$ & 53077.3 & 149.9 & 0.067 & 0.038 & $v$ \\
\hline 834.2 & 9.9 & 13.718 & 0.903 & $v$ & 71192.4 & 18.0 & 0.069 & 0.121 & $v$ \\
\hline 1066.4 & 9.9 & 19.918 & 1.096 & $v$ & 80344.4 & 149.9 & 0.004 & 0.035 & $v$ \\
\hline 1241.8 & 9.9 & 18.517 & 1.053 & $v$ & 80648.3 & 149.9 & 0.047 & 0.037 & $v$ \\
\hline 1415.6 & 9.9 & 11.828 & 0.837 & $v$ & 111203.5 & 169.5 & 0.063 & 0.036 & $v$ \\
\hline 1588.3 & 9.9 & 11.398 & 0.820 & $v$ & 122914.8 & 158.1 & 0.038 & 0.035 & $v$ \\
\hline 1761.6 & 9.9 & 10.388 & 0.788 & $v$ & 128677.6 & 310.6 & 0.049 & 0.026 & $v$ \\
\hline 5576.8 & 99.9 & 1.641 & 0.115 & $v$ & 134477.4 & 288.0 & 0.013 & 0.026 & $v$ \\
\hline 7013.2 & 99.9 & 1.123 & 0.101 & $v$ & 157567.5 & 309.5 & 0.018 & 0.025 & $v$ \\
\hline 16773.0 & 149.9 & 0.338 & 0.051 & $v$ & 163345.1 & 309.6 & 0.015 & 0.024 & $v$ \\
\hline 17076.6 & 149.9 & 0.302 & 0.050 & $v$ & 169123.9 & 308.7 & 0.018 & 0.025 & $v$ \\
\hline 17380.0 & 149.9 & 0.337 & 0.051 & $v$ & 192030.6 & 102.3 & 0.039 & 0.045 & $v$ \\
\hline 41428.4 & 149.9 & 0.048 & 0.037 & $v$ & 203748.6 & 264.3 & 0.050 & 0.028 & $v$ \\
\hline 41731.9 & 149.9 & 0.082 & 0.039 & $v$ & 221119.4 & 312.7 & 0.016 & 0.024 & $v$ \\
\hline 42035.4 & 149.9 & 0.126 & 0.041 & $v$ & 232682.9 & 304.9 & 0.013 & 0.025 & $v$ \\
\hline 52470.2 & 149.9 & 0.053 & 0.037 & $v$ & $\ldots$ & $\ldots$ & $\ldots$ & $\ldots$ & $v$ \\
\hline 587.4 & 9.9 & 6.547 & 0.659 & $b$ & 75259.9 & 149.9 & 0.114 & 0.050 & $b$ \\
\hline 760.2 & 9.9 & 20.774 & 1.144 & $b$ & 87035.9 & 149.9 & 0.116 & 0.050 & $b$ \\
\hline 1164.5 & 9.9 & 33.987 & 1.501 & $b$ & 87339.4 & 149.9 & 0.052 & 0.048 & $b$ \\
\hline 1341.7 & 9.9 & 28.043 & 1.347 & $b$ & 87642.9 & 149.9 & 0.034 & 0.047 & $b$ \\
\hline 1514.1 & 9.9 & 21.021 & 1.154 & $b$ & 99662.9 & 88.9 & 0.012 & 0.060 & $b$ \\
\hline 1686.7 & 9.9 & 18.354 & 1.076 & $b$ & 110054.4 & 322.4 & 0.075 & 0.034 & $b$ \\
\hline 1860.6 & 9.9 & 15.713 & 1.035 & $b$ & 116707.9 & 147.4 & 0.024 & 0.047 & $b$ \\
\hline 6397.2 & 99.9 & 2.714 & 0.148 & $b$ & 122031.6 & 237.9 & 0.098 & 0.040 & $b$ \\
\hline 10993.9 & 149.9 & 0.958 & 0.093 & $b$ & 127387.6 & 322.4 & 0.051 & 0.032 & $b$ \\
\hline 11297.6 & 149.9 & 1.020 & 0.094 & $b$ & 133277.3 & 299.9 & 0.056 & 0.033 & $b$ \\
\hline 11601.7 & 149.9 & 1.102 & 0.095 & $b$ & 139026.1 & 305.9 & 0.038 & 0.033 & $b$ \\
\hline 23604.9 & 149.9 & 0.332 & 0.059 & $b$ & 144734.8 & 319.4 & 0.059 & 0.033 & $b$ \\
\hline 23908.6 & 149.9 & 0.405 & 0.061 & $b$ & 150570.4 & 308.4 & 0.026 & 0.033 & $b$ \\
\hline 24212.1 & 149.9 & 0.359 & 0.060 & $b$ & 156280.2 & 321.9 & 0.057 & 0.033 & $b$ \\
\hline 30146.5 & 149.9 & 0.265 & 0.056 & $b$ & 162058.5 & 321.4 & 0.047 & 0.033 & $b$ \\
\hline 30449.9 & 149.9 & 0.308 & 0.058 & $b$ & 167840.3 & 320.9 & 0.019 & 0.032 & $b$ \\
\hline 30676.6 & 73.1 & 0.192 & 0.085 & $b$ & 190956.6 & 319.9 & 0.086 & 0.034 & $b$ \\
\hline 34763.7 & 149.9 & 0.194 & 0.054 & $b$ & 196608.1 & 144.9 & 0.009 & 0.046 & $b$ \\
\hline 35067.2 & 149.9 & 0.063 & 0.049 & $b$ & 202511.7 & 319.9 & 0.003 & 0.031 & $b$ \\
\hline 35370.8 & 149.9 & 0.167 & 0.053 & $b$ & 209280.3 & 120.9 & 0.045 & 0.053 & $b$ \\
\hline
\end{tabular}


Table 1

(Continued)

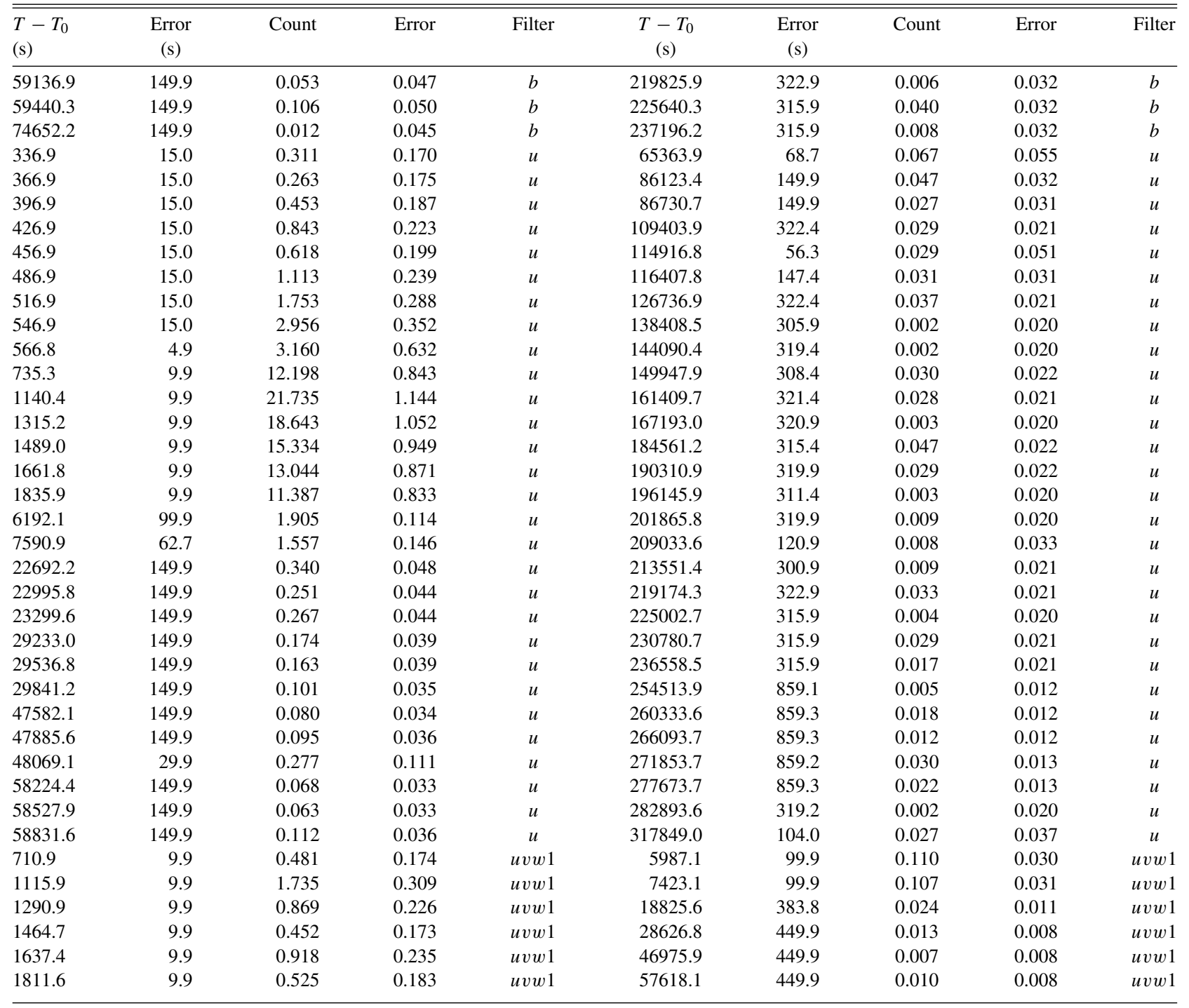

except for the first point that was observed in the $v$ band but has been normalized to white using the late-time UVOT data. The UVOT light curve shows only two major peaks. The first small peak (146-180 s) shows weak correlation with the BAT. After $\sim 40 \mathrm{~s}$, it re-brightens to its second and brightest peak at $209 \mathrm{~s}$, coinciding with the brightest $\gamma$-ray peak in the BAT light curve. Overall, the optical data are smoother and trace the BAT data better than the XRT data.

Several vertical lines shown in Figure 1 partition the light curve into nine different intervals according to the UVOT significance criterion to obtain time-resolved joint-instrument spectral analysis using the XRT, the BAT, and the WAM data. Since the prompt emission of GRB 110205A is observed by multiple instruments, the systematic uncertainty among the instruments to perform the joint analysis must be carefully understood.

The energy response function of XRT has been examined by the observations of supernova remnants and active galactic nuclei with various X-ray missions such as Suzaku and $X M M$-Newton. According to the simultaneous observation of Cyg X-1 between XRT (WT mode) and Suzaku/XIS, ${ }^{42}$ the pho-

42 http://swift.gsfc.nasa.gov/docs/heasarc/caldb/swift/docs/xrt/
SWIFT-XRT-CALDB-09_v16.pdf ton index and the observed flux agree within $\sim 5 \%$ and $\sim 15 \%$, respectively. The spectral calibration of BAT has been based on Crab Nebula observations at various boresight angles. The photon index and the flux are within $\sim 5 \%$ and $\sim 10 \%$ of the assumed Crab values based on Rothschild et al. (1998) and Jung (1989). Similarly, the WAM energy response has been investigated using the Crab spectrum collected by the Earth occultation technique (Sakamoto et al. 2011a). The spectral shape and its normalization are consistent within 10\%-15\% with the result of the INTEGRAL SPI instrument (Sizun et al. 2004). The crossinstrument calibration between BAT and WAM has been investigated deeply by Sakamoto et al. (2011b) using simultaneously observed bright GRBs. According to this work, the normalization of the BAT-WAM joint fit agrees within $10 \%-15 \%$ to the BAT data. The cross-instrument calibration between XRT (WT mode) and BAT has been investigated by the simultaneous observation of Cyg X-1. Both the spectral shape and the flux agree within the $5 \%-10 \%$ range between XRT and BAT. ${ }^{43}$ In summary, based on the single instrument and the cross-instrument calibration effort, the systematic uncertainty among XRT, BAT,

\footnotetext{
43 The presentation in the 2009 Swift conference: http://www.swift.psu.edu/swift-2009/.
} 
Table 2

Photomertric Observations for GRB 110205A from Ground-based Telescopes

\begin{tabular}{|c|c|c|c|c|c|c|c|c|c|}
\hline $\begin{array}{l}T-T_{0} \\
\text { (s) }\end{array}$ & $\begin{array}{l}\text { Exp } \\
(\mathrm{s})\end{array}$ & Mag & Error & Filter & $\begin{array}{c}T-T_{0} \\
\quad \text { (s) }\end{array}$ & $\begin{array}{l}\text { Exp } \\
\text { (s) }\end{array}$ & Mag & Error & Filter \\
\hline \multicolumn{10}{|c|}{ ROTSE-IIIb } \\
\hline 135.5 & 107.0 & 17.79 & 0.42 & $C$ & 1695.7 & 60.0 & 14.84 & 0.06 & $C$ \\
\hline 340.2 & 282.2 & 16.42 & 0.11 & $C$ & 1765.0 & 60.0 & 14.89 & 0.08 & $C$ \\
\hline 520.5 & 60.0 & 16.33 & 0.20 & $C$ & 1834.2 & 60.0 & 15.04 & 0.09 & $C$ \\
\hline 589.6 & 60.0 & 15.75 & 0.12 & $C$ & 1903.0 & 60.0 & 15.01 & 0.07 & $C$ \\
\hline 658.4 & 60.0 & 15.25 & 0.09 & $C$ & 1971.8 & 60.0 & 15.22 & 0.08 & $C$ \\
\hline 727.5 & 60.0 & 14.81 & 0.06 & $C$ & 2040.7 & 60.0 & 15.28 & 0.08 & $C$ \\
\hline 796.6 & 60.0 & 14.50 & 0.06 & $C$ & 2109.5 & 60.0 & 15.23 & 0.07 & $C$ \\
\hline 865.7 & 60.0 & 14.27 & 0.05 & $C$ & 2178.4 & 60.0 & 15.44 & 0.11 & $C$ \\
\hline 935.1 & 60.0 & 14.19 & 0.04 & $C$ & 2247.2 & 60.0 & 15.40 & 0.11 & $C$ \\
\hline 1004.3 & 60.0 & 14.08 & 0.03 & $C$ & 2384.7 & 60.0 & 15.51 & 0.11 & $C$ \\
\hline 1073.5 & 60.0 & 14.06 & 0.03 & $C$ & 2453.9 & 60.0 & 15.46 & 0.11 & $C$ \\
\hline 1142.7 & 60.0 & 14.14 & 0.05 & $C$ & 2660.7 & 60.0 & 15.71 & 0.11 & $C$ \\
\hline 1211.9 & 60.0 & 14.18 & 0.04 & $C$ & 2868.0 & 60.0 & 15.83 & 0.12 & $C$ \\
\hline 1281.0 & 60.0 & 14.31 & 0.06 & $C$ & 2937.2 & 60.0 & 16.02 & 0.13 & $C$ \\
\hline 1350.3 & 60.0 & 14.40 & 0.06 & $C$ & 3075.3 & 60.0 & 15.82 & 0.15 & $C$ \\
\hline 1419.5 & 60.0 & 14.53 & 0.05 & $C$ & 3144.5 & 60.0 & 15.96 & 0.15 & $C$ \\
\hline 1489.1 & 60.0 & 14.53 & 0.05 & $C$ & 3213.4 & 60.0 & 16.00 & 0.13 & $C$ \\
\hline 1558.0 & 60.0 & 14.55 & 0.06 & $C$ & 3593.1 & 680.0 & 16.15 & 0.05 & $C$ \\
\hline 1626.6 & 60.0 & 14.67 & 0.06 & $C$ & $\ldots$ & $\ldots$ & $\ldots$ & $\ldots$ & - \\
\hline
\end{tabular}

\begin{tabular}{|c|c|c|c|c|c|c|c|c|c|}
\hline \multicolumn{10}{|c|}{ LOT (AB magnitude) } \\
\hline 39647.6 & 180.0 & 20.95 & 0.07 & $g^{\prime}$ & 43061.7 & 300.0 & 19.49 & 0.06 & $z^{\prime}$ \\
\hline 42062.9 & 300.0 & 21.05 & 0.05 & $g^{\prime}$ & 39856.7 & 180.0 & 20.32 & 0.05 & $r^{\prime}$ \\
\hline 45217.0 & 600.0 & 20.93 & 0.03 & $g^{\prime}$ & 42392.9 & 300.0 & 20.47 & 0.04 & $r^{\prime}$ \\
\hline 48824.2 & 600.0 & 20.90 & 0.02 & $g^{\prime}$ & 45846.0 & 600.0 & 20.35 & 0.04 & $r^{\prime}$ \\
\hline 52111.7 & 600.0 & 21.07 & 0.03 & $g^{\prime}$ & 49453.2 & 600.0 & 20.42 & 0.03 & $r^{\prime}$ \\
\hline 55478.8 & 600.0 & 21.25 & 0.03 & $g^{\prime}$ & 52740.7 & 600.0 & 20.51 & 0.02 & $r^{\prime}$ \\
\hline 58774.9 & 600.0 & 21.33 & 0.04 & $g^{\prime}$ & 56108.6 & 600.0 & 20.70 & 0.03 & $r^{\prime}$ \\
\hline 62047.7 & 600.0 & 21.44 & 0.04 & $g^{\prime}$ & 59404.8 & 600.0 & 20.80 & 0.03 & $r^{\prime}$ \\
\hline 65421.7 & 600.0 & 21.55 & 0.04 & $g^{\prime}$ & 62677.6 & 600.0 & 20.97 & 0.04 & $r^{\prime}$ \\
\hline 40064.1 & 180.0 & 19.97 & 0.06 & $i^{\prime}$ & 66052.4 & 600.0 & 20.98 & 0.03 & $r^{\prime}$ \\
\hline 42720.4 & 300.0 & 20.19 & 0.05 & $i^{\prime}$ & 68767.1 & 600.0 & 21.08 & 0.04 & $r^{\prime}$ \\
\hline 46474.1 & 600.0 & 20.11 & 0.03 & $i^{\prime}$ & 69377.1 & 600.0 & 21.10 & 0.04 & $r^{\prime}$ \\
\hline 50079.6 & 600.0 & 20.13 & 0.03 & $i^{\prime}$ & 69987.9 & 600.0 & 21.22 & 0.05 & $r^{\prime}$ \\
\hline 53368.0 & 600.0 & 20.31 & 0.03 & $i^{\prime}$ & 129844.0 & 600.0 & 22.50 & 0.17 & $r^{\prime}$ \\
\hline 56735.0 & 600.0 & 20.45 & 0.04 & $i^{\prime}$ & 130563.0 & 600.0 & 22.54 & 0.15 & $r^{\prime}$ \\
\hline 60032.0 & 600.0 & 20.62 & 0.04 & $i^{\prime}$ & 131174.0 & 600.0 & 22.36 & 0.11 & $r^{\prime}$ \\
\hline 63304.9 & 600.0 & 20.69 & 0.05 & $i^{\prime}$ & 133798.0 & 600.0 & 22.47 & 0.09 & $r^{\prime}$ \\
\hline 66678.8 & 600.0 & 20.81 & 0.04 & $i^{\prime}$ & 134409.0 & 600.0 & 22.38 & 0.09 & $r^{\prime}$ \\
\hline 40287.9 & 180.0 & 19.68 & 0.09 & $z^{\prime}$ & 135019.0 & 600.0 & 22.60 & 0.12 & $r^{\prime}$ \\
\hline \multicolumn{10}{|c|}{ HCT } \\
\hline 67639.0 & 1080.0 & 21.00 & 0.04 & $R$ & 72379.0 & 600.0 & 21.15 & 0.04 & $R$ \\
\hline 69019.0 & 540.0 & 21.00 & 0.05 & $R$ & $\ldots$ & $\ldots$ & $\ldots$ & $\ldots$ & - \\
\hline \multicolumn{10}{|c|}{$0.61 \mathrm{~m}$ Lightbuckets } \\
\hline 20747.0 & 300.0 & 18.67 & 0.02 & $C$ & 27549.9 & 300.0 & 19.19 & 0.08 & $R$ \\
\hline \multicolumn{10}{|c|}{ GRAS 005} \\
\hline 3536.8 & 600.0 & 15.98 & 0.02 & $C$ & 4248.7 & 600.0 & 16.44 & 0.02 & $R$ \\
\hline 5327.4 & 300.0 & 16.51 & 0.02 & $C$ & 5857.5 & 600.0 & 16.89 & 0.03 & $R$ \\
\hline 6902.9 & 600.0 & 17.02 & 0.03 & $C$ & 6378.8 & 300.0 & 17.10 & 0.04 & $R$ \\
\hline 8366.9 & 300.0 & 17.33 & 0.04 & $C$ & 7992.8 & 300.0 & 17.62 & 0.08 & $R$ \\
\hline \multicolumn{10}{|c|}{$1.5 \mathrm{~m} \mathrm{OSN}$} \\
\hline 10152.2 & 300.0 & 18.93 & 0.03 & $B$ & 12747.2 & 90.0 & 18.15 & 0.02 & $R$ \\
\hline 11527.2 & 300.0 & 19.07 & 0.03 & $B$ & 13337.9 & 90.0 & 18.18 & 0.03 & $R$ \\
\hline 12426.7 & 150.0 & 19.17 & 0.03 & $B$ & 14361.8 & 90.0 & 18.34 & 0.03 & $R$ \\
\hline 13091.5 & 150.0 & 19.21 & 0.03 & $B$ & 14856.1 & 90.0 & 18.32 & 0.03 & $R$ \\
\hline 14115.7 & 150.0 & 19.32 & 0.03 & $B$ & 85598.9 & 3300.0 & 20.89 & 0.05 & $R$ \\
\hline 14610.0 & 150.0 & 19.38 & 0.04 & $B$ & 10489.4 & 300.0 & 18.67 & 0.06 & $U$ \\
\hline 76574.1 & 400.0 & 21.87 & 0.17 & $B$ & 11871.4 & 500.0 & 18.96 & 0.06 & $U$ \\
\hline 9871.3 & 240.0 & 17.22 & 0.02 & $I$ & 13592.3 & 500.0 & 19.22 & 0.06 & $U$ \\
\hline 11278.2 & 240.0 & 17.41 & 0.02 & $I$ & 9644.8 & 180.0 & 18.04 & 0.02 & V \\
\hline 12840.2 & 200.0 & 17.59 & 0.02 & $I$ & 11094.4 & 180.0 & 18.35 & 0.02 & $V$ \\
\hline
\end{tabular}


Table 2

(Continued)

\begin{tabular}{|c|c|c|c|c|c|c|c|c|c|}
\hline $\begin{array}{l}T-T_{0} \\
\text { (s) }\end{array}$ & $\begin{array}{l}\operatorname{Exp} \\
\text { (s) }\end{array}$ & Mag & Error & Filter & $\begin{array}{c}T-T_{0} \\
\text { (s) }\end{array}$ & $\begin{array}{l}\text { Exp } \\
\text { (s) }\end{array}$ & Mag & Error & Filter \\
\hline 13430.8 & 120.0 & 17.65 & 0.03 & $I$ & 12653.7 & 90.0 & 18.55 & 0.02 & V \\
\hline 14454.7 & 120.0 & 17.77 & 0.03 & $I$ & 13244.3 & 90.0 & 18.61 & 0.03 & $V$ \\
\hline 14949.0 & 120.0 & 17.78 & 0.03 & $I$ & 14268.5 & 90.0 & 18.71 & 0.04 & V \\
\hline 77630.9 & 300.0 & 20.56 & 0.19 & $I$ & 14762.8 & 90.0 & 18.66 & 0.04 & $V$ \\
\hline 9409.4 & 180.0 & 17.59 & 0.02 & $R$ & 76977.2 & 350.0 & 21.68 & 0.19 & $V$ \\
\hline \multicolumn{10}{|c|}{$1 \mathrm{~m} \mathrm{LOAO}$} \\
\hline 13101.0 & 720.0 & 19.32 & 0.16 & $B$ & 12846.0 & 180.0 & 18.16 & 0.03 & $R$ \\
\hline 11974.0 & 180.0 & 18.14 & 0.03 & $R$ & 13273.0 & 180.0 & 18.22 & 0.03 & $R$ \\
\hline 12416.0 & 180.0 & 18.11 & 0.03 & $R$ & $\ldots$ & $\ldots$ & $\ldots$ & $\ldots$ & - \\
\hline \multicolumn{10}{|c|}{ BOOTES-1 } \\
\hline 219.4 & 117.0 & 17.43 & 0.38 & $C$ & 2715.2 & 49.0 & 15.66 & 0.07 & $C$ \\
\hline 1603.5 & 48.0 & 14.61 & 0.05 & $C$ & 2832.0 & 64.0 & 15.74 & 0.07 & $C$ \\
\hline 2118.2 & 48.0 & 15.18 & 0.05 & $C$ & 3096.5 & 197.0 & 15.81 & 0.04 & $C$ \\
\hline 2218.1 & 48.5 & 15.29 & 0.05 & $C$ & 3709.1 & 413.0 & 16.21 & 0.04 & $C$ \\
\hline 2317.5 & 48.0 & 15.45 & 0.06 & $C$ & 4621.4 & 495.5 & 16.54 & 0.05 & $C$ \\
\hline 2417.5 & 48.5 & 15.44 & 0.06 & $C$ & 5615.5 & 495.5 & 16.88 & 0.08 & $C$ \\
\hline 2516.8 & 48.0 & 15.35 & 0.05 & $C$ & 6658.3 & 545.0 & 17.15 & 0.08 & $C$ \\
\hline 2615.8 & 48.0 & 15.51 & 0.07 & $C$ & 8066.6 & 859.5 & 17.59 & 0.10 & $C$ \\
\hline \multicolumn{10}{|c|}{ BOOTES-2 } \\
\hline 939.3 & 5.0 & 14.40 & 0.04 & $R$ & 6300.8 & 363.5 & 17.19 & 0.04 & $R$ \\
\hline 994.7 & 5.0 & 14.24 & 0.04 & $R$ & 7149.9 & 484.5 & 17.42 & 0.04 & $R$ \\
\hline 2015.1 & 72.5 & 15.26 & 0.05 & $R$ & 8362.6 & 727.0 & 17.65 & 0.04 & $R$ \\
\hline 2375.1 & 63.0 & 15.63 & 0.08 & $R$ & 9818.1 & 969.0 & 17.98 & 0.05 & $R$ \\
\hline 5120.9 & 189.5 & 16.67 & 0.04 & $R$ & 11758.0 & 969.5 & 18.16 & 0.06 & $R$ \\
\hline 5373.4 & 187.0 & 16.83 & 0.04 & $R$ & 13940.7 & 1212.0 & 18.52 & 0.08 & $R$ \\
\hline 5748.5 & 187.0 & 17.10 & 0.05 & $R$ & $\ldots$ & $\ldots$ & $\ldots$ & $\ldots$ & - \\
\hline \multicolumn{10}{|c|}{$1.23 \mathrm{~m} \mathrm{Calar}$ Alto } \\
\hline 2467.0 & 60.0 & 15.59 & 0.06 & $R$ & 9692.0 & 60.0 & 17.88 & 0.08 & $R$ \\
\hline 2582.0 & 60.0 & 15.64 & 0.06 & $R$ & 10202.0 & 60.0 & 17.91 & 0.05 & $R$ \\
\hline 4198.0 & 60.0 & 16.48 & 0.05 & $R$ & 10712.0 & 60.0 & 18.02 & 0.05 & $R$ \\
\hline 4661.0 & 60.0 & 16.60 & 0.04 & $R$ & 11215.0 & 60.0 & 18.05 & 0.05 & $R$ \\
\hline 5107.0 & 60.0 & 16.73 & 0.05 & $R$ & 11336.0 & 60.0 & 18.10 & 0.05 & $R$ \\
\hline 5559.0 & 60.0 & 16.85 & 0.05 & $R$ & 11835.0 & 60.0 & 18.15 & 0.05 & $R$ \\
\hline 6008.0 & 60.0 & 16.99 & 0.05 & $R$ & 12337.0 & 60.0 & 18.26 & 0.06 & $R$ \\
\hline 6461.0 & 60.0 & 17.05 & 0.05 & $R$ & 12844.0 & 60.0 & 18.26 & 0.07 & $R$ \\
\hline 6909.0 & 60.0 & 17.23 & 0.04 & $R$ & 13353.0 & 60.0 & 18.28 & 0.05 & $R$ \\
\hline 7358.0 & 60.0 & 17.41 & 0.05 & $R$ & 13905.0 & 60.0 & 18.34 & 0.05 & $R$ \\
\hline 7804.0 & 60.0 & 17.49 & 0.05 & $R$ & 14018.0 & 60.0 & 18.41 & 0.05 & $R$ \\
\hline 8670.0 & 60.0 & 17.73 & 0.05 & $R$ & 14128.0 & 60.0 & 18.35 & 0.05 & $R$ \\
\hline 9182.0 & 60.0 & 17.75 & 0.05 & $R$ & 14239.0 & 60.0 & 18.45 & 0.06 & $R$ \\
\hline \multicolumn{10}{|c|}{$2.2 \mathrm{~m}$ Calar Alto } \\
\hline 9475.0 & 100.0 & 17.67 & 0.03 & $R$ & 12388.0 & 500.0 & 18.13 & 0.03 & $R$ \\
\hline 10064.0 & 500.0 & 17.76 & 0.03 & $R$ & 12962.0 & 500.0 & 18.18 & 0.03 & $R$ \\
\hline 10639.0 & 500.0 & 17.88 & 0.03 & $R$ & 13535.0 & 500.0 & 18.26 & 0.03 & $R$ \\
\hline 11214.0 & 500.0 & 17.97 & 0.03 & $R$ & 14109.0 & 500.0 & 18.33 & 0.03 & $R$ \\
\hline 11787.0 & 500.0 & 18.04 & 0.03 & $R$ & 14706.0 & 500.0 & 18.41 & 0.03 & $R$ \\
\hline 15281.0 & 500.0 & 18.49 & 0.03 & $R$ & $\ldots$ & $\ldots$ & $\ldots$ & $\ldots$ & - \\
\hline \multicolumn{10}{|c|}{$1.6 \mathrm{~m} \mathrm{AZT-33IK}$} \\
\hline 37988.0 & 3060.0 & 19.97 & 0.08 & $R$ & 62132.0 & 6600.0 & 20.94 & 0.07 & $R$ \\
\hline \multicolumn{10}{|c|}{ Zeiss-600 } \\
\hline 794.0 & 60.0 & 14.47 & 0.04 & $R$ & 2006.2 & 60.0 & 15.26 & 0.06 & $\bar{V}$ \\
\hline 853.6 & 60.0 & 14.18 & 0.03 & $R$ & 2068.4 & 60.0 & 15.55 & 0.06 & $V$ \\
\hline 918.4 & 60.0 & 14.08 & 0.02 & $R$ & 2128.0 & 60.0 & 15.52 & 0.06 & $V$ \\
\hline 1022.1 & 60.0 & 14.03 & 0.02 & $R$ & 2230.0 & 2.0 & 15.27 & 0.05 & $R$ \\
\hline 1081.7 & 60.0 & 14.00 & 0.02 & $R$ & 2350.9 & 2.0 & 15.46 & 0.05 & $R$ \\
\hline 1142.2 & 60.0 & 14.07 & 0.02 & $R$ & 2742.3 & 2.0 & 15.54 & 0.06 & $R$ \\
\hline 1204.4 & 60.0 & 14.09 & 0.02 & $R$ & 3133.7 & 2.0 & 15.92 & 0.09 & $R$ \\
\hline 1264.0 & 60.0 & 14.34 & 0.02 & $R$ & 3284.9 & 2.0 & 16.06 & 0.08 & $R$ \\
\hline 1323.7 & 60.0 & 14.29 & 0.03 & $R$ & 3436.1 & 2.0 & 16.08 & 0.04 & $R$ \\
\hline 1385.9 & 60.0 & 14.37 & 0.02 & $R$ & 3557.9 & 2.0 & 16.11 & 0.04 & $R$ \\
\hline
\end{tabular}


Table 2

(Continued)

\begin{tabular}{|c|c|c|c|c|c|c|c|c|c|}
\hline $\begin{array}{l}T-T_{0} \\
\text { (s) }\end{array}$ & $\begin{array}{c}\text { Exp } \\
\text { (s) }\end{array}$ & Mag & Error & Filter & $\begin{array}{c}T-T_{0} \\
\text { (s) }\end{array}$ & $\begin{array}{c}\text { Exp } \\
\text { (s) }\end{array}$ & Mag & Error & Filter \\
\hline 1446.3 & 60.0 & 14.48 & 0.03 & $R$ & 3678.9 & 2.0 & 16.04 & 0.06 & $R$ \\
\hline 1506.0 & 60.0 & 14.52 & 0.03 & $R$ & 3799.9 & 2.0 & 16.16 & 0.05 & $R$ \\
\hline 1568.2 & 60.0 & 14.59 & 0.03 & $R$ & 3921.7 & 2.0 & 16.19 & 0.05 & $R$ \\
\hline 1627.8 & 60.0 & 14.58 & 0.03 & $R$ & 4042.7 & 2.0 & 16.19 & 0.05 & $R$ \\
\hline 1688.3 & 60.0 & 14.74 & 0.03 & $R$ & 4163.6 & 2.0 & 16.30 & 0.06 & $R$ \\
\hline 1886.1 & 60.0 & 15.16 & 0.09 & $V$ & 4588.7 & 4.0 & 16.44 & 0.05 & $R$ \\
\hline 1945.7 & 60.0 & 15.38 & 0.04 & V & 4830.6 & 4.0 & 16.59 & 0.06 & $R$ \\
\hline
\end{tabular}

and WAM should be within $15 \%$ in both the spectral shape and its normalization of the spectrum. To accommodate systematic uncertainties, we include a multiplication factor in the range $0.85-1.15$ for the flux normalization for each instrument.

We first applied the spectral analysis to the time-averaged interval B (intB; see Figure 1 for interval definition) and find that the photon index in a simple power-law model derived by the individual instrument differs significantly. The photon indices derived by the XRT, the BAT, and the WAM spectra are $-1.12 \pm 0.03,-1.71 \pm 0.04$, and $-2.27_{-0.27}^{+0.22}$, respectively (also listed in Table 3; spectral fitting errors in Table 3 and in this section are given in $90 \%$ confidence). Since these differences are significantly larger than the systematic uncertainty associated with the instrumental cross-calibration (the systematic error in the photon index is \pm 0.3 for the worst case as discussed above), the apparent change of spectral slope is very likely intrinsic to the GRB. Thus, the observed broadband spectrum requires two breaks to connect the XRT, the BAT, and the WAM data.

According to the GRB synchrotron emission model, the overall spectrum should be a broken power law characterized by several break frequencies (e.g., the self-absorption frequency $v_{a}$, the cooling frequency $v_{c}$, and the frequency of minimum electron injection energy $v_{m}$ ). However, the well-known Band function, which only includes one break energy, cannot represent the shape of the more complex spectrum of this particular event; therefore, we extended the analysis code, Xspec, to include two additional spectral functions. The first one is a double "Band" spectrum with two spectral breaks, which was labeled bkn2band:

$$
f(E)=\left\{\begin{array}{l}
A E^{\alpha} \exp \left(-E / E_{0}\right), \quad \text { if } E \leqslant \frac{E_{0} E_{1}}{E_{1}-E_{0}}(\alpha-\beta) \\
A\left[\frac{E_{0} E_{1}}{E_{1}-E_{0}}(\alpha-\beta)\right]^{\alpha-\beta} \exp (\beta-\alpha) E^{\beta} \exp \left(-E / E_{1}\right), \\
\quad \text { if } \frac{E_{0} E_{1}}{E_{1}-E_{0}}(\alpha-\beta)<E \leqslant(\beta-\gamma) E_{1} \\
A\left[\frac{E_{0} E_{1}}{E_{1}-E_{0}}(\alpha-\beta)\right]^{\alpha-\beta} \exp (\beta-\alpha) . \\
{\left[(\beta-\gamma) E_{1}\right]^{\beta-\gamma} \exp (\gamma-\beta) E^{\gamma},} \\
\quad \text { if } E>(\beta-\gamma) E_{1}
\end{array}\right.
$$

where $A$ is the normalization at $1 \mathrm{keV}$ in units of photons $\mathrm{cm}^{-2} \mathrm{~s}^{-1} \mathrm{keV}^{-1} ; \alpha, \beta$, and $\gamma$ are the photon indices of the three power-law segments; and $E_{0}$ and $E_{1}$ are the two break energies. However, when fitting the spectrum using this new $b k n 2 b a n d$ model, the third power-law index, $\gamma$, is poorly constrained mainly due to the poor statistics in the high-energy WAM data above $400 \mathrm{keV}$. For this reason, the second new model, bandcut, replaces the third power-law component with an exponential cutoff: 44

$$
f(E)= \begin{cases}A E^{\alpha} \exp \left(-E / E_{0}\right), & \text { if } E \leqslant \frac{E_{0} E_{1}}{E_{1}-E_{0}}(\alpha-\beta) \\ A\left[\frac{E_{0} E_{1}}{E_{1}-E_{0}}(\alpha-\beta)\right]^{\alpha-\beta} & \exp (\beta-\alpha) E^{\beta} \exp \left(-E / E_{1}\right) \\ & \text { if } E>\frac{E_{0} E_{1}}{E_{1}-E_{0}}(\alpha-\beta) .\end{cases}
$$

Note that the exponential cutoff in the new bandcut model introduces a second break, E1, although different from the break in a doubly broken power-law model or $b k n 2 b a n d$ model. The new bandcut model can well characterize the prompt emission spectra of GRB 110205A. Hereafter, we use "two-break energy spectrum" to represent the bandcut model spectrum. Figure 2 shows the XRT, BAT, and WAM joint fit spectral analysis of intB based on the bandcut model and the Band function fit. The systematic residuals from the best-fit Band function are evident especially in the WAM data. The best-fit parameters based on the bandcut model are $\alpha=-0.50_{-0.08}^{+0.09}, E_{0}=5.0_{-0.8}^{+1.1} \mathrm{keV}$, $\beta=-1.54_{-0.09}^{+0.10}$, and $E_{1}=333_{-118}^{+265} \mathrm{keV}\left(\chi^{2} / \mathrm{dof}=529.8 / 503\right)$. On the other hand, the best-fit parameters based on the Band function are $\alpha=-0.59_{-0.08}^{+0.06}, \beta=-1.72_{-0.03}^{+0.01}$, and $E_{\text {peak }}=$ $9.5_{-0.9}^{+1.5} \mathrm{keV}\left(\chi^{2} /\right.$ dof $\left.=575.7 / 504\right)$. Therefore, $\Delta \chi^{2}\left(\equiv \chi_{\text {Band }}^{2}-\right.$ $\left.\chi_{\text {bandcut }}^{2}\right)$ between the Band function and the bandcut model is 45.9 for 1 dof. To quantify the significance of this improvement, we performed 10,000 spectral simulations assuming the bestfit Band function parameters by folding the energy response functions and the background data of XRT, BAT, and WAM. Then, we determine in how many cases the bandcut model fit gives $\chi^{2}$ improvements equal to or greater than $\Delta \chi^{2}=45.9$ for 1 dof over the Band function fit. We found equal or higher improvements in none of the simulated spectra out of 10,000 . Thus, the chance probability of having an equal or higher $\Delta \chi^{2}$ of 45.9 with the bandcut when the parent distribution is actually the Band function is $<0.01 \%$. A caveat for this simulation is that the statistical improvement of the joint fit may be not as high as this simulation indicates if the calibration uncertainties among the instruments are included.

The same method is then applied to perform the joint spectral fitting to the remaining time intervals. Table 3 shows the bestfit results from the bandcut model. This is the first time that two spectral breaks are clearly identified in the prompt GRB spectra. The two breaks are consistent with the expectation of the broken power-law synchrotron spectrum (see discussion in Section 4.1).

\footnotetext{
44 Both bkn2band and bandcut new models can be downloaded from the following Web site: http://asd.gsfc.nasa.gov/Takanori.Sakamoto/personal/. They can be used to fit future GRBs with similar characteristics.
} 

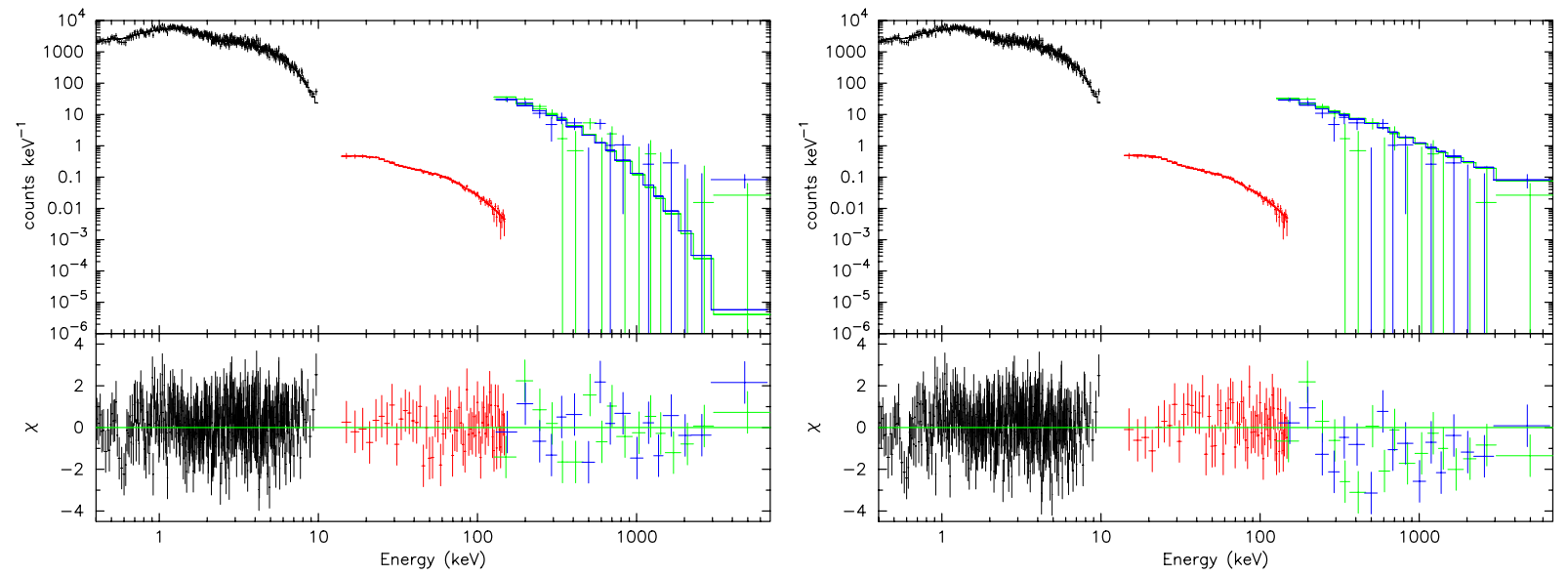

Figure 2. XRT (black), BAT (red), and WAM (green and blue) count spectra of intB with the bandcut model (left panel) and the standard Band function (right panel) fit. The improvement using the bandcut model can be easily seen in the residual panel at the bottom.

(A color version of this figure is available in the online journal.)

Table 3

Best Spectral Fit Result from XRT, BAT, and WAM Joint Fitting with the bandcut Model

\begin{tabular}{|c|c|c|c|c|c|c|c|c|c|c|}
\hline Int & $t_{1}$ & $t_{2}$ & $\alpha$ & $E_{0}$ & $\beta$ & $E_{1}$ & $\chi^{2} /$ dof & XRT & BAT & WAM \\
\hline int1 & 146 & 155 & $-0.40_{-0.25}^{+0.43}$ & $4.9_{-2.5}^{+4.7}$ & $-1.45_{-0.21}^{+0.13}$ & $207_{-88}^{+468}$ & $126.4 / 137=0.92$ & $-1.05_{-0.11}^{+0.11}$ & $-1.68_{-0.09}^{+0.09}$ & $-2.24_{-0.70}^{+0.48}$ \\
\hline int2 & 164 & 194 & $-0.22_{-0.14}^{+0.15}$ & $4.4_{-0.9}^{+1.4}$ & $-1.61_{-0.19}^{+0.11}$ & $258_{-59}^{+615}$ & $269.7 / 256=1.05$ & $-0.94_{-0.06}^{+0.05}$ & $-1.81_{-0.06}^{+0.06}$ & $-2.72_{-0.84}^{+0.63}$ \\
\hline int3 & 194 & 204 & $-0.41_{-0.21}^{+0.31}$ & $5.5_{-2.4}^{+4.5}$ & $-1.44_{-0.14}^{+0.13}$ & $264_{-115}^{+336}$ & $138.5 / 145=0.96$ & $-0.99_{-0.11}^{+0.10}$ & $-1.62_{-0.08}^{+0.08}$ & $-2.14_{-0.98}^{+0.57}$ \\
\hline int4 & 204 & 214 & $-0.26_{-0.36}^{+0.37}$ & $4.1_{-1.8}^{+8.2}$ & $-1.26_{-0.08}^{+0.11}$ & $299_{-96}^{+175}$ & $107.6 / 146=0.74$ & $-0.91_{-0.10}^{+0.09}$ & $-1.45_{-0.06}^{+0.05}$ & $-2.22_{-0.25}^{+0.20}$ \\
\hline int5 & 214 & 224 & $-0.37_{-0.26}^{+0.31}$ & $5.8_{-2.5}^{+7.6}$ & $-1.39_{-0.11}^{+0.13}$ & $273_{-96}^{+189}$ & $144.5 / 144=1.00$ & $-0.87_{-0.10}^{+0.09}$ & $-1.58_{-0.06}^{+0.06}$ & $-2.42_{-0.43}^{+0.43}$ \\
\hline int6 & 224 & 234 & $-0.34_{-0.24}^{+0.25}$ & $4.4_{-1.6}^{+3.1}$ & $-1.53_{-0.11}^{+0.15}$ & $289_{-135}^{+750}$ & $146.9 / 142=1.03$ & $-1.00_{-0.10}^{+0.09}$ & $-1.72_{-0.08}^{+0.08}$ & $-1.98_{-0.63}^{+0.38}$ \\
\hline int7 & 234 & 284 & $-0.61_{-0.14}^{+0.16}$ & $4.7_{-1.3}^{+2.3}$ & $-1.65_{-0.13}^{+0.07}$ & $342_{-156}^{+958}$ & $248.8 / 233=1.07$ & $-1.24_{-0.06}^{+0.06}$ & $-1.78_{-0.08}^{+0.08}$ & $-2.00_{-1.16}^{+0.55}$ \\
\hline $\operatorname{int} A$ & 164 & 254 & $-0.32_{-0.10}^{+0.10}$ & $4.7_{-0.8}^{+1.1}$ & $-1.52_{-0.07}^{+0.10}$ & $346_{-115}^{+229}$ & $430.9 / 437=0.99$ & $-1.00_{-0.04}^{+0.03}$ & $-1.68_{-0.04}^{+0.04}$ & $-2.28_{-0.24}^{+0.19}$ \\
\hline intB & 164 & 314 & $-0.50_{-0.08}^{+0.09}$ & $5.0_{-0.8}^{+1.1}$ & $-1.54_{-0.09}^{+0.10}$ & $333_{-118}^{+265}$ & $529.8 / 503=1.05$ & $-1.12_{-0.03}^{+0.03}$ & $-1.71_{-0.04}^{+0.04}$ & $-2.27_{-0.27}^{+0.22}$ \\
\hline
\end{tabular}

Note. The last three columns show the photon index from each single instrument fitting. Errors are given in $90 \%$ confidence.

Figure 3 shows the evolution of different parameters from the time-resolved spectral fitting between $146 \mathrm{~s}$ and $284 \mathrm{~s}$. The two break energies, $E_{0}$ and $E_{1}$, remain almost constant during this time range, with $E_{0} \sim 5 \mathrm{keV}$ and $E_{1} \sim 300 \mathrm{keV}$. However, the large errors for $E_{0}$ and $E_{1}$ prevent us from drawing a firmer conclusion regarding the temporal evolution of the two break energies. The low-energy photon index, $\alpha$, also shows no statistically significant evolution. However, the high-energy photon index, $\beta$, does show a weak evolution: it becomes slightly harder during the brightest BAT peak around $210 \mathrm{~s}$. Over all, the $\alpha$ value during the time range is around -0.35 and $\beta$ is around -1.5 . The peak energy derived from interval B (intB) is $E_{p}=$ $(2+\beta) E_{1}=153_{-54}^{+121} \mathrm{keV}$.

Although the UVOT optical data are not included in the spectral fitting, they are shown in the best-fit spectra in Figure 4. For all the intervals, the optical data are above the extrapolation of the best spectral fits for high energies. A speculation may be that the observed $\alpha$ is somehow harder than the expected value of the synchrotron model. However, even if we set $\alpha$ to the predicted value, $-2 / 3$, the optical data are still above the best-fit spectra in some intervals. This suggests that the optical emission may have a different origin from the high-energy emission (see Section 4 for more detailed discussion).

\subsection{Afterglow Analysis}

\subsubsection{Light Curves}

Shortly after the prompt emission, the optical light curve is characterized by a surprisingly steep rise and a bright peak around $1100 \mathrm{~s}$. The early steep rise, starting around $350 \mathrm{~s}$, is observed by both ROTSE and UVOT, and the peak, which is wide and smooth, reaches $R=14.0 \mathrm{mag}$ at $1073 \mathrm{~s}$ after the burst. Such a bright peak around this time with such a steep rise is rare and unusual (Oates et al. 2009; Panaitescu \& Vestrand 2011) and has only been reported in a few cases (e.g., Volnova et al. 2010; Nardini et al. 2011). A peak brightness of $R=$ 14.0 mag at $\sim 1100 \mathrm{~s}$ after the burst ranks the optical afterglow as one of the brightest ever observed in this same time range (Akerlof \& Swan 2007). Following the peak, the light curve decays monotonically, as shown in Figure 5, and displays a slight flattening around $3000 \mathrm{~s}$. Around $5 \times 10^{4} \mathrm{~s}$, there is a rebrightening feature observed in the $g^{\prime}, r^{\prime}, i^{\prime}, z^{\prime}$ bands by LOT (Figure 6), and a final steepening is observed after $\sim 10^{5} \mathrm{~s}$. Overall, no substantial color evolution is observed in the optical band.

The X-ray data show a different temporal behavior (see also Figure 5). Shortly after the prompt emission, the light curve has a very steep decay, between $350 \mathrm{~s}$ and $700 \mathrm{~s}$. This is followed by a small re-brightening bump around $1100 \mathrm{~s}$, the peak time of the optical light curve, and a monotonic decay afterward. There might be a late X-ray flare around $5000 \mathrm{~s}$, but the lack of X-ray data just before $\sim 5000 \mathrm{~s}$ prevents any robust conclusion from being drawn.

The light curves were fit with one or the superposition of two broken power-law functions. The broken power-law function has been widely adopted to fit afterglow light curves with both the rising and decay phases (e.g., Rykoff et al. 2009) and works 


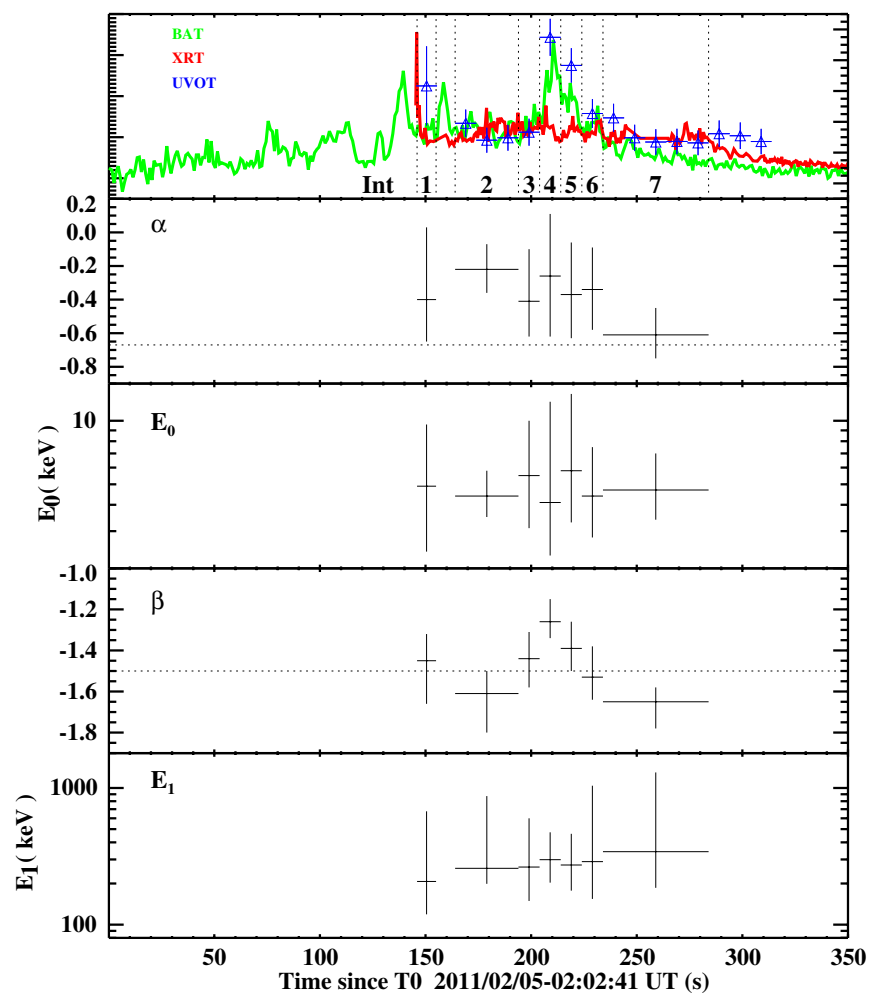

Figure 3. Time evolution of different parameters from the time-resolved spectral fitting of GRB 110205A. Top panel has the same key as Figure 1. The two horizontal dotted lines in $\alpha$ and $\beta$ panels are the predicted synchrotron photon index value for fast cooling phase from the standard GRB fireball model. Errors are given at $90 \%$ confidence except the top panel.

(A color version of this figure is available in the online journal.)

well for most cases (e.g., Liang et al. 2010). The function can be represented as

$$
f=\left(\frac{t}{t_{b}}\right)^{\alpha_{1}}\left[1+\left(\frac{t}{t_{b}}\right)^{s(\alpha 1-\alpha 2)}\right]^{-1 / s},
$$

where $f$ is the flux, $t_{b}$ is the break time, $\alpha 1$ and $\alpha 2$ are the two power-law indices before and after the break, and $s$ is a smoothing parameter. According to this definition, the peak time $t_{p}$, where the flux reaches maximum, is

$$
t_{p}=t_{b}\left(\frac{\alpha 1}{-\alpha 2}\right)^{1 / s(\alpha 1-\alpha 2)}
$$

If a multiple broken power-law function is required to present more than one break time, Equation (2) in van Eerten \& MacFadyen (2011) is adopted. We first tried one broken powerlaw component and found that it could not fully represent the feature near the optical peak, mainly because of its unusually late and steep rising feature, which was not observed in previous GRBs, and a slight flattening feature around $3000 \mathrm{~s}$ after the peak shown in $R$ band. Noticing that there is a peak in both the optical and the X-ray light curves around $1100 \mathrm{~s}$ and that the optical light curve flattens around $3000 \mathrm{~s}$, we speculate that there is a significant contribution of emission from the RS. An RS contribution to the X-ray band has not been well identified in the past. Theoretically, the RS synchrotron emission peaks around the optical band so that its synchrotron extension to the $\mathrm{X}$-ray band is expected to be weak. In any case, under certain conditions, it is possible that the RS synchrotron (Fan \& Wei

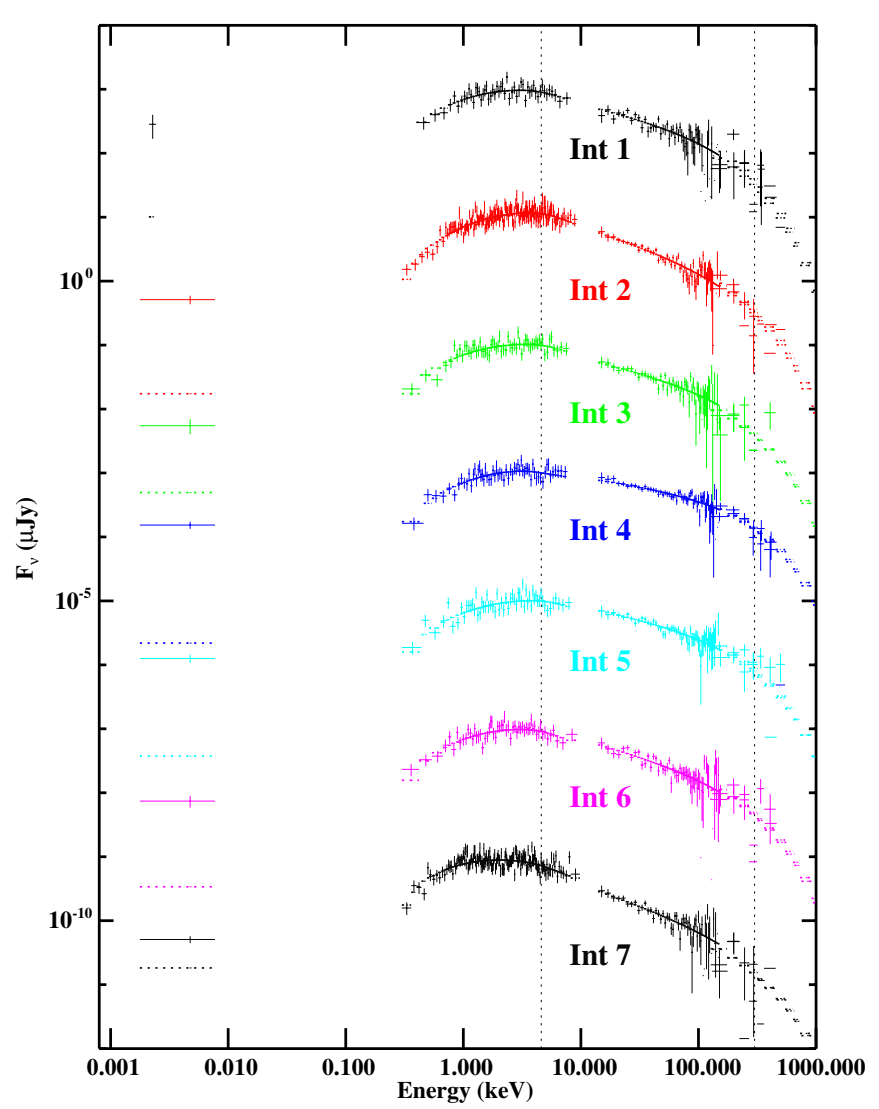

Figure 4. Best-fit prompt spectra of GRB 110205A, different colors present different intervals (see Figure 1 for interval definition). Note that WAM data above $400 \mathrm{keV}$ are not shown in the figure due to large uncertainties when transforming counts to flux density in Xspec above this energy range. Intervals 2-7 are shifted by a factor of $10^{-2}$ accumulatively for the purpose of clarity. UVOT optical data are not included during the fitting, but they are shown in the best-fit spectrum. Solid lines present the observed data, while dashed lines present the prediction of the best-fit band cut model. The two vertical lines show the mean value of $E_{0}$ and $E_{1}$ from the fitting.

(A color version of this figure is available in the online journal.)

2005; Zou et al. 2005) or SSC (Kobayashi et al. 2007) emission would contribute significantly to the X-ray band to create a bump feature. In order to account for both the FS and the RS components, we fit both the optical and X-ray light curves with the superposition of two broken power-law functions. For the $\mathrm{X}$-ray light curve, an additional single power-law component was applied for the steep decay phase, as usually seen in Swift afterglows (e.g., Tagliaferri et al. 2005).

Two light-curve models have been adopted. The standard afterglow model predicts that the blast wave enters the deceleration phase as the RS crosses the ejecta (Sari \& Piran 1995; Zhang et al. 2003). If the FS $v_{m}$ is already below the optical band at the crossing time, both the FS and the RS would have the same peak time $\left(t_{p}\right)$ in the optical band. This defines our first scenario, in which the optical peak is generated by both the RS and the FS. If, however, $v_{m}$ is initially above the optical band at the deceleration time, the FS optical light curve would display a rising feature ( $\propto t^{1 / 2}$; Sari et al. 1998) initially, until reaching a peak at a later time when $v_{m}$ crosses the optical band. This is our second scenario. In this case, the early optical peak is mostly dominated by the RS component. This is the Type I light curve identified in Zhang et al. (2003) and Jin \& Fan (2007). We now discuss the two scenarios in turn.

Scenario I. We performed a simultaneous fit to both optical $R$-band data and X-ray data by setting the same break time in the 

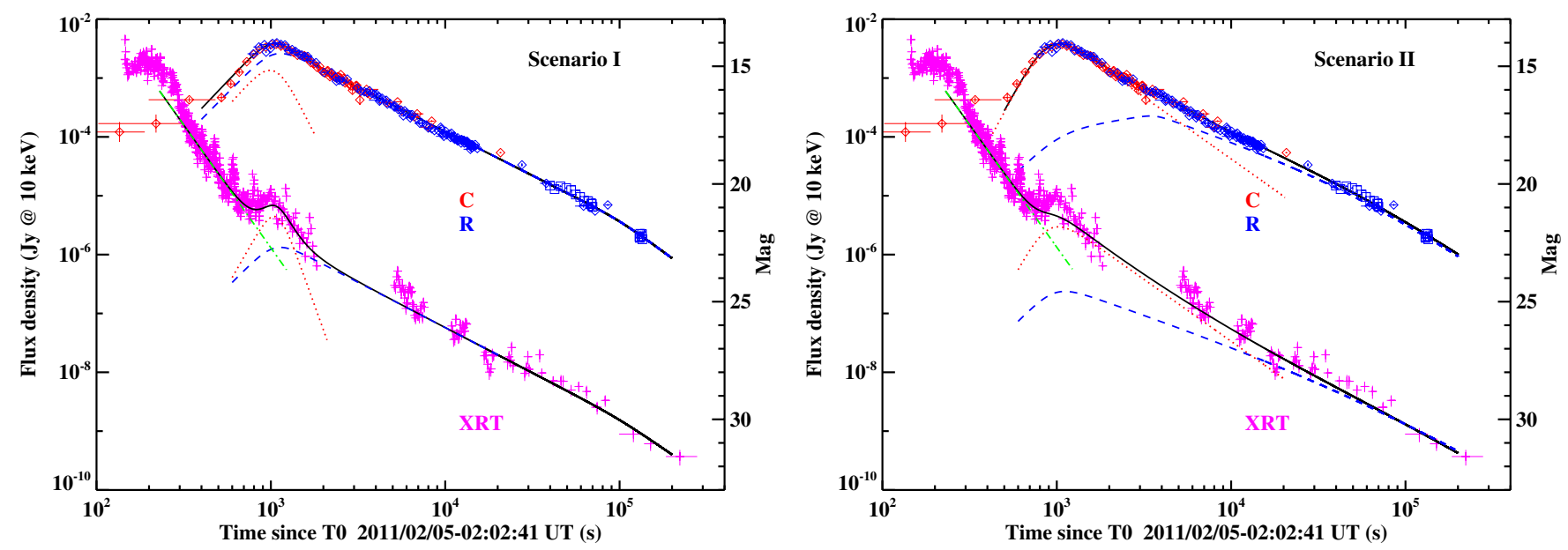

Figure 5. GRB 110205A light curves from XRT data (pink cross) and optical data, including $C$ band (red diamond) and $R$ band (blue square). Two scenarios are considered. Both scenarios consider superposition of FS (dashed line) and RS (dotted line) contribution. The main difference is that Scenario I (left panel) considers the optical peak as dominated by both FS and RS combination while Scenario II considers the optical peak as dominated by RS only. The dot-dashed line represents the steep decay phase for X-ray data. The solid line represents the combination of all components.

(A color version of this figure is available in the online journal.)
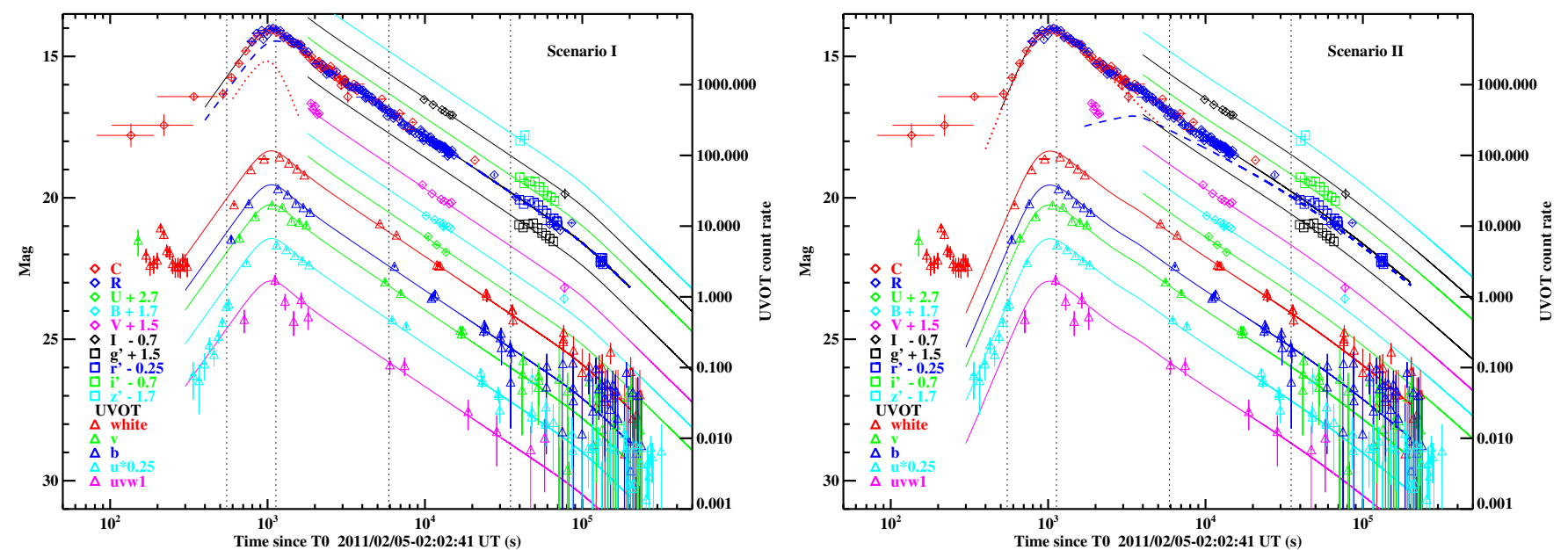

Figure 6. Optical light curves of GRB 110205A re-scaled from $R$-band best fit. The early fast rise behavior in optical band can be better explained by Scenario II (right panel) than Scenario I (left panel).

(A color version of this figure is available in the online journal.)

two bands. A late time break is invoked to fit both the $R$-band and X-ray light curves. However, we exclude the re-brightening feature around $5 \times 10^{4} \mathrm{~s}$ in the optical band and the steep decay phase of X-rays before $400 \mathrm{~s}$, which is likely the tail of the prompt emission (Zhang et al. 2006). For the FS component, the rising temporal index is fixed to be 3 based on the slow cooling interstellar medium (ISM) model during the pre-deceleration phase $^{45}$ (e.g., Xue et al. 2009; Shen \& Matzner 2012). The rising slope of the RS component is left as a free parameter to be constrained from the data. The best simultaneous fit results are summarized in Table 4 and shown in Figure 5 (left panel). The red dot and blue dashed lines represent the RS and FS components, respectively. The black solid line is the sum of all the components.

Next we apply the same best $R$-band fit parameters and rescale to the optical data in other bands. The results are shown in Figure 6 (left panel). Light curves in different bands are properly

\footnotetext{
45 In the coasting phase of a thin shell decelerated by a constant density medium, the forward shock has the scalings $v_{m} \propto t^{0}, v_{c} \propto t^{-2}$, and $F_{v, m} \propto t^{3}$. For $v_{m}<v_{\mathrm{opt}}<v_{c}$, one has $F_{v} \propto t^{3}$.
}

shifted for clarity. As we can see, the model quite adequately describes most light curves. The only exception is the UVOT- $u$ band, in which the model curve overpredicts the early-time flux between $300 \mathrm{~s}$ and $600 \mathrm{~s}$.

Scenario II. In this scenario, the bright peak around $1100 \mathrm{~s}$ and the steep rise phase at early times in the optical band are contributed by the RS only. The FS component shows up later. According to the afterglow model, the FS component is characterized by a double broken power law with a rising index +3 before the deceleration time ( $t_{p}$ of the RS), +0.5 before the FS peak, and a normal decay (decay index to be fit from the data) after the FS peak (e.g., Sari et al. 1998; Zhang et al. 2003; Xue et al. 2009). For the RS component, the rising index is fixed to be 5.5, which is the mean value of a single power-law fit to the $R$-band data $(\alpha \sim 5)$ and the UVOT- $u$ band data $(\alpha \sim 6)$ only. In the X-ray band, we still use a similar model as Scenario I with a superposition of an RS and an FS component. The model parameters are not well constrained, especially for the $\mathrm{X}$-ray peak around $1100 \mathrm{~s}$ due to its narrow peak. We tried to fix several parameters in order to reach an acceptable fitting for this scenario. The best simultaneous fit results for both $R$-band 
Table 4

Best-fit Result from $R$-band and X-Ray Light Curves

\begin{tabular}{|c|c|c|c|c|}
\hline \multirow[b]{2}{*}{ Par } & \multicolumn{2}{|c|}{ Scenario I } & \multicolumn{2}{|c|}{ Scenario II } \\
\hline & Optical & X-ray & Optical & X-ray \\
\hline FS & & & FS & \\
\hline$\alpha_{f 1}$ & $3^{\mathrm{a}}$ & $3^{\mathrm{a}}$ & $3^{\mathrm{a}} \rightarrow 0.5^{\mathrm{a}}$ & $3^{\mathrm{a}}$ \\
\hline$t_{p}(\mathrm{~s})$ & $1064 \pm 42$ & $1064^{b}$ & $3.64 \pm 1.0 \times 10^{3}$ & $1021 \pm 26$ \\
\hline$F_{p}(\mathrm{Jy})$ & $\begin{array}{c}4.96 \times 10^{-3} \\
14.48 \mathrm{mag}\end{array}$ & $1.32 \times 10^{-6}$ & $\begin{array}{c}4.35 \times 10^{-4} \\
17.12 \mathrm{mag}\end{array}$ & $1.42 \times 10^{-7}$ \\
\hline$\alpha_{f 2}$ & $-1.50 \pm 0.04$ & $-1.54 \pm 0.10$ & $-1.01 \pm 0.01$ & $-1.00^{\mathrm{a}}$ \\
\hline$t_{j b}(\mathrm{~s})$ & $1.0 \pm 0.2 \times 10^{5}$ & $1.0 \times 10^{5 b}$ & $5.44 \pm 0.2 \times 10^{4}$ & $5.44 \times 10^{4 b}$ \\
\hline$\alpha_{f 3}$ & $-2.18 \pm 0.8$ & $-2.05 \pm 0.5$ & $-2.05 \pm 0.7$ & $-1.75 \pm 1.0$ \\
\hline RS & & & RS & \\
\hline $\begin{array}{l}\alpha_{r 1} \\
t_{p}(\mathrm{~s})\end{array}$ & $\begin{array}{c}3.32 \pm 1.2 \\
1064^{\mathrm{b}}\end{array}$ & $\begin{array}{c}5.19 \pm 1.3 \\
1064^{b}\end{array}$ & $\begin{array}{c}5.5^{\mathrm{a}} \\
1021^{\mathrm{b}}\end{array}$ & $\begin{array}{c}5.5^{\mathrm{a}} \\
1021^{\mathrm{b}}\end{array}$ \\
\hline$F_{p}(\mathrm{Jy})$ & $\begin{array}{c}2.47 \times 10^{-3} \\
15.24 \mathrm{mag}\end{array}$ & $4.40 \times 10^{-6}$ & $\begin{array}{c}7.19 \times 10^{-3} \\
14.07 \mathrm{mag}\end{array}$ & $3.59 \times 10^{-6}$ \\
\hline$\alpha_{r 2}$ & $-5.90 \pm 1.0$ & $\begin{array}{l}-8.26 \pm 1.3 \\
\text { Steep decay }\end{array}$ & $-2.10^{\mathrm{a}}$ & $\begin{array}{c}-2.10^{\mathrm{a}} \\
\text { Steep decay }\end{array}$ \\
\hline$\alpha_{\mathrm{sd}}$ & & $-4.18 \pm 0.2$ & & $-4.16 \pm 0.2$ \\
\hline
\end{tabular}

Notes.

a This indicates that the parameter is fixed during the fitting.

b This indicates that the parameter is simultaneously fit for both optical and $\mathrm{X}$-ray data.

and X-ray band data are also summarized in Table 4 and shown in Figure 5 (right panel). Similar to Scenario I, we then used the same model and parameters derived from the $R$-band fit rescaled to other light curves. The results are shown in Figure 6 (right panel), again with shifting. This scenario can well explain the early fast rise behavior in all bands.

Comparing the two scenarios, we find that Scenario I can represent most optical data and can better account for the X-ray data than Scenario II. However, it cannot well match the early very steep rise in the UVOT- $u$ band. On the other hand, Scenario II can represent the early fast rising behavior in the optical band (including UVOT- $u$ band), but the fits to the X-ray data are not as good as Scenario I. We note that our Scenario II is similar to another variant of Scenario II recently proposed by Gao (2011), but we conclude that the optical data before $\sim$ $350 \mathrm{~s}$ are generated by the optical prompt emission.

\subsubsection{Afterglow SED Analysis}

In order to study the SED of the afterglow, we selected four epochs when we have the best multi-band data coverage: $550 \mathrm{~s}$, $1.1 \mathrm{ks}, 5.9 \mathrm{ks}$, and $35 \mathrm{ks}$. Since no significant color evolution is observed in the optical data, we interpolate (or extrapolate if necessary) the optical band light curve to these epochs when no direct observations are available at the epochs. The optical data used for constructing the SED are listed in Table 5. The X-ray data are re-binned using the data around the four epochs. The spectral fitting is then performed using Xspec software. During the fitting, the Galactic hydrogen column density, $N_{\mathrm{H}}$, is fixed to $1.6 \times 10^{20} \mathrm{~cm}^{-2}$ (Kalberla et al. 2005), and the host galaxy hydrogen column density is fixed to $4.0 \times 10^{21} \mathrm{~cm}^{-2}$. These are derived from an average spectral fitting of the late-time XRT PC data. We tried both the broken power-law and the single powerlaw models for the afterglow SED. For the broken power-law model, we set $\Gamma_{2}=\Gamma_{1}-0.5$, assuming a cooling break between the optical and X-ray bands. Meanwhile, we also investigated
Table 5

SED Data (in magnitude) at Four Different Epochs

\begin{tabular}{lcccc}
\hline \hline Time & $550 \mathrm{~s}$ & $1.13 \mathrm{ks}$ & $5.9 \mathrm{ks}$ & $35 \mathrm{ks}$ \\
\hline$u v w 1$ & $20.13 \pm 0.50$ & $16.91 \pm 0.18$ & $19.71 \pm 0.30$ & $22.50 \pm 0.70$ \\
$u$ & $17.13 \pm 0.20$ & $14.98 \pm 0.06$ & $17.50 \pm 0.10$ & $20.47 \pm 0.30$ \\
$b$ & $17.54 \pm 0.16$ & $15.23 \pm 0.05$ & $17.92 \pm 0.07$ & $20.97 \pm 0.30$ \\
$v$ & $17.13 \pm 0.30$ & $14.68 \pm 0.06$ & $17.45 \pm 0.14$ & $20.63 \pm 0.50$ \\
white & $17.80 \pm 0.12$ & $15.25 \pm 0.05$ & $17.92 \pm 0.05$ & $20.74 \pm 0.22$ \\
$U$ & $\ldots$ & $\ldots$ & $17.74 \pm 0.1$ & $20.69 \pm 0.1$ \\
$B$ & $\ldots$ & $\ldots$ & $18.05 \pm 0.1$ & $20.86 \pm 0.1$ \\
$V$ & $\ldots$ & $\ldots$ & $17.24 \pm 0.1$ & $20.20 \pm 0.1$ \\
$R$ & $16.07 \pm 0.20$ & $14.10 \pm 0.06$ & $16.84 \pm 0.1$ & $19.73 \pm 0.1$ \\
$I$ & $\ldots$ & $\ldots$ & $16.39 \pm 0.1$ & $19.21 \pm 0.1$ \\
$g$ & $\ldots$ & $\ldots$ & $\ldots$ & $20.76 \pm 0.07$ \\
$r$ & $\ldots$ & $\ldots$ & $\ldots$ & $20.12 \pm 0.05$ \\
$i$ & $\ldots$ & $\ldots$ & $\ldots$ & $19.76 \pm 0.06$ \\
$z$ & $\ldots$ & & & $19.46 \pm 0.09$ \\
\hline
\end{tabular}

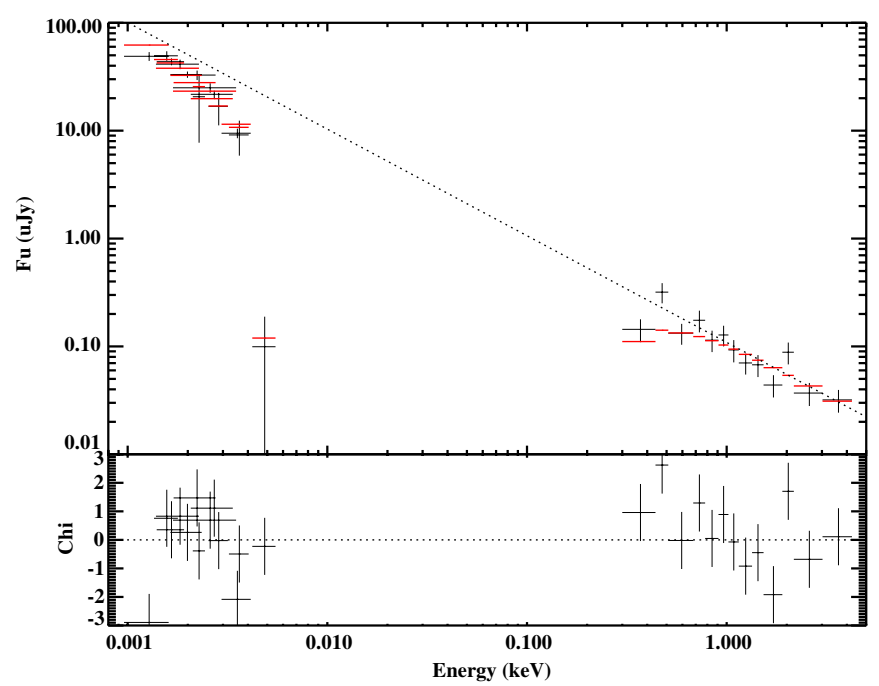

Figure 7. Afterglow SED of GRB $110205 \mathrm{~A}$ at $35 \mathrm{ks}$. Black points are the observed data; red points are the predicted value from the model. Dotted line is the original power-law model without absorption or extinction.

(A color version of this figure is available in the online journal.)

the use of three different extinction laws, namely, the Milky Way (MW) and, Large and Small Magellanic Clouds (LMC and $\mathrm{SMC})$, for the host galaxy extinction model. The $E(B-V)$ from the Galactic extinction is set to 0.01 (Schlegel et al. 1998) during the fitting. The best-fit results are listed in Table 6 .

The $5.9 \mathrm{ks}$ and $35 \mathrm{ks}$ (see Figure 7) SEDs have the best data coverage, and they are ascribable to the FS component only. We therefore use these two SEDs to constrain the extinction properties of the afterglow. We find that the SMC and LMC dust models provide acceptable and better fits than the MW dust model. The data are equally well fit by the broken powerlaw and the single power-law model. For the broken power-law model, the break energy is found to be within the optical band $(0.0025 \pm 0.0006 \mathrm{keV})$. Within the $3 \sigma$ error, one cannot separate the optical and X-ray data to two different spectral regimes. The lack of clear breaks in optical light curves between 5.9 and 35 ks also disfavors the possibility of the break energy passing through the optical band.

\subsection{Host Galaxy Search}

We have performed a deep search for the host galaxy of the GRB. Observations were performed with the $2.2 \mathrm{~m}$ Calar Alto 
Table 6

SED Fitting at Different Epochs

\begin{tabular}{|c|c|c|c|c|c|c|c|}
\hline Model & Time & $E(B-V)$ & $\Gamma_{1}$ & $E_{b}(\mathrm{keV})$ & $\Gamma_{2}=\Gamma_{1}+0.5$ & Norm & $\chi^{2} /$ dof \\
\hline \multirow[t]{5}{*}{$\overline{\mathrm{MW}}$} & Bknplaw & & & & & & \\
\hline & $550 \mathrm{~s}$ & $0.11_{-0.02}^{+0.02}$ & $1.36_{-0.034}^{+0.033}$ & $0.41_{-0.107}^{+0.074}$ & 1.86 & $0.23_{-0.019}^{+0.039}$ & $124.8 / 81=1.54$ \\
\hline & $1.13 \mathrm{ks}$ & $0.19_{-0.004}^{+0.009}$ & $2.10_{-0.022}^{+0.021}$ & $4.94_{-4.93}^{+4.93}$ & 2.60 & $0.038_{-4.0 E-3}^{+4.39 E-3}$ & $13.6 / 4=3.6$ \\
\hline & $5.9 \mathrm{ks}$ & $0.16_{-0.007}^{+0.02}$ & $1.59_{-0.027}^{+0.0100}$ & $1.00 \mathrm{E}-03_{-1.0 E-3}^{+1.5 E-3}$ & 2.09 & $0.086_{-0.022}^{+4.34 E-3}$ & $20.2 / 23=0.88$ \\
\hline & $35 \mathrm{ks}$ & $0.13_{-0.008}^{+0.009}$ & $1.57_{-0.012}^{+0.011}$ & $\begin{array}{r}1.70 \mathrm{E}-03_{-1.57 E-4}^{+3.66 E-3} \\
\end{array}$ & 2.07 & $4.03 \mathrm{E}-03_{-1.89 E-3}^{+2.15 E-4}$ & $38.9 / 22=1.77$ \\
\hline \multirow[t]{5}{*}{ MW } & plaw & & & & & & \\
\hline & $550 \mathrm{~s}$ & 0.26 & $\ldots$ & $\ldots$ & 1.70 & 0.14 & $179.8 / 82=2.19$ \\
\hline & $1.13 \mathrm{ks}$ & $0.19_{-0.009}^{+0.009}$ & $\ldots$ & $\ldots$ & $2.10_{-0.022}^{+0.022}$ & $0.038_{-0.004}^{+0.004}$ & $13.6 / 5=2.72$ \\
\hline & $5.9 \mathrm{ks}$ & $0.16_{-0.019}^{+0.020}$ & $\ldots$ & $\ldots$ & $2.09_{-0.027}^{+0.027}$ & $0.027_{-1 E-4}^{+1 E-4}$ & $20.2 / 24=0.84$ \\
\hline & $35 \mathrm{ks}$ & 0.13 & $\ldots$ & $\ldots$ & 2.07 & $1.59 \mathrm{E}-04$ & $55.6 / 23=2.42$ \\
\hline \multirow[t]{5}{*}{ LMC } & Bknplaw & & & & & & \\
\hline & $550 \mathrm{~s}$ & $0.10_{-0.017}^{+0.021}$ & $1.39_{-0.033}^{+0.033}$ & $0.36_{-0.151}^{+0.0951}$ & 1.89 & $0.25_{-0.027}^{+0.076}$ & $116.1 / 81=1.43$ \\
\hline & $1.13 \mathrm{ks}$ & $0.16_{-0.004}^{+0.013}$ & $1.64_{-0.030}^{+0.013}$ & $2.88_{3.34 E-3}^{+2.60 E-3}$ & 2.14 & $0.72_{-0.027}^{+0.035}$ & $8.5 / 4=2.13$ \\
\hline & $5.9 \mathrm{ks}$ & $0.13_{-0.019}^{+0.018}$ & $1.61_{-0.049}^{+0.099}$ & $2.87 \mathrm{E}-03_{-9.33 E-4}^{+7.01 E-4}$ & 2.11 & $0.051_{-0.020}^{+0.007}$ & $16.1 / 23=0.70$ \\
\hline & $35 \mathrm{ks}$ & $0.10_{-0.007}^{+0.006}$ & $1.55_{-0.014}^{+0.016}$ & $2.07 \mathrm{E}-03_{-3.48 E-4}^{+5.14 E-4}$ & 2.05 & $3.70 \mathrm{E}-03_{-3.85 E-4}^{+2.58 E-4}$ & $26.4 / 22=1.20$ \\
\hline \multirow[t]{5}{*}{ LMC } & plaw & & & & & & \\
\hline & $550 \mathrm{~s}$ & $0.24_{-0.021}^{+0.023}$ & $\cdots$ & $\cdots$ & $1.75_{-0.031}^{+0.033}$ & $0.141_{-3.85 E-3}^{+3.93 E-3}$ & $162.5 / 82=1.98$ \\
\hline & $1.13 \mathrm{ks}$ & $0.14_{-0.006}^{+0.006}$ & $\ldots$ & $\ldots$ & $2.09_{-0.021}^{+0.021}$ & $0.039_{-4.13 E-3}^{+4.45 E-3}$ & $13.9 / 5=2.78$ \\
\hline & $5.9 \mathrm{ks}$ & $0.10_{-0.011}^{+0.011}$ & $\ldots$ & $\ldots$ & $2.04_{-0.020}^{+0.020}$ & $2.71 \mathrm{E}-03_{-1.35 E-4}^{+0.001}$ & $19.4 / 24=0.81$ \\
\hline & $35 \mathrm{ks}$ & $0.07_{-0.009}^{+0.006}$ & $\cdots$ & $\cdots$ & $2.01_{-0.015}^{+0.015}$ & $\begin{array}{r}1.65 \mathrm{E}-04_{-1.05 E-05}^{+1.04 E-05} \\
\end{array}$ & $39.0 / 23=1.70$ \\
\hline \multirow[t]{5}{*}{ SMC } & Bknplaw & & & & & & \\
\hline & $550 \mathrm{~s}$ & $0.08_{-0.014}^{+0.016}$ & $1.38_{-0.032}^{+0.032}$ & $0.37_{-0.14}^{+0.09}$ & 1.88 & $0.25_{-0.026}^{+0.068}$ & $114.3 / 81=1.41$ \\
\hline & $1.13 \mathrm{ks}$ & $0.11_{-0.005}^{+0.005}$ & $2.06_{-0.021}^{+0.020}$ & $5.05_{-4.93}^{+-4.93}$ & 2.56 & $0.039_{-2.24 E-3}^{+4.56 E-3}$ & $24.7 / 4=6.18$ \\
\hline & $5.9 \mathrm{ks}$ & $0.09_{-0.004}^{+0.005}$ & $1.56_{-0.028}^{+0.024}$ & $2.74 \mathrm{E}-03_{-5.05 E-4}^{+9.4 E-4}$ & 2.06 & $0.052_{-7.14 E-3}^{+5.57 E-3}$ & $17.3 / 23=0.75$ \\
\hline & $35 \mathrm{ks}$ & $0.08_{-0.006}^{+0.005}$ & $1.54_{-0.013}^{+0.016}$ & $2.43 \mathrm{E}-03_{-3.7 E-4}^{+4.53 E-4}$ & 2.04 & $3.42 \mathrm{E}-03_{-2.75 E-4}^{+2.78 E-4}$ & $24.3 / 22=1.1$ \\
\hline \multirow[t]{5}{*}{ SMC } & plaw & & & & & & \\
\hline & $550 \mathrm{~s}$ & 0.19 & $\ldots$ & $\ldots$ & 1.75 & 0.136 & $189.5 / 82=2.31$ \\
\hline & $1.13 \mathrm{ks}$ & $0.11_{-0.005}^{+0.005}$ & $\cdots$ & $\cdots$ & $2.06_{-0.020}^{+0.021}$ & $0.039_{-4.12 E-3}^{+4.40 E-3}$ & $24.7 / 5=4.14$ \\
\hline & $5.9 \mathrm{ks}$ & $0.07_{-0.007}^{+0.007}$ & $\cdots$ & $\cdots$ & $2.01_{-0.017}^{+0.017}$ & $2.68 \mathrm{E}-03_{-1.3 E-4}^{+1.3 E-4}$ & $22.5 / 24=0.94$ \\
\hline & $35 \mathrm{ks}$ & $0.05_{-0.006}^{+0.005}$ & $\cdots$ & $\cdots$ & $1.98_{-0.013}^{+0.014}$ & $\begin{array}{r}1.65 \mathrm{E}-04_{-1.04 E-05}^{+1.03 E-05} \\
\end{array}$ & $36.7 / 23=1.60$ \\
\hline
\end{tabular}

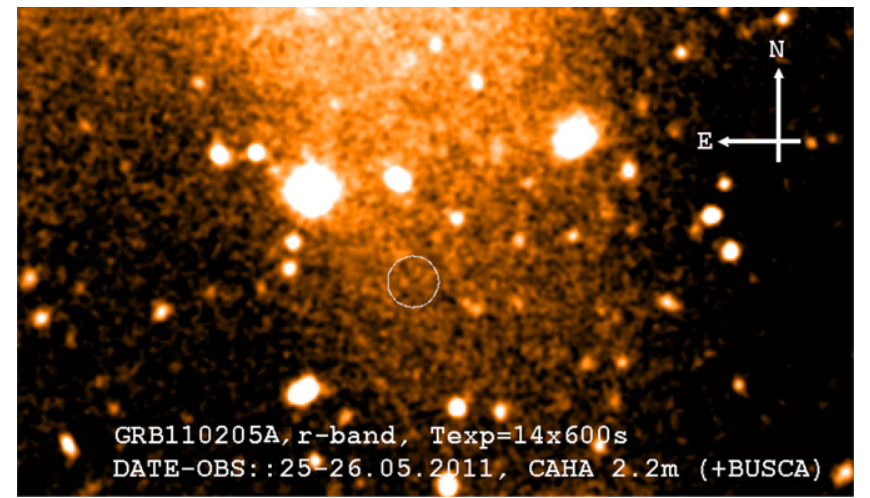

Figure 8. Host galaxy searching from $2.2 \mathrm{~m}$ Calar Alto telescope taken in $r^{\prime}$ band. The center of the circle indicates the afterglow location. No clear source is detected down to $r^{\prime} \sim 24.8$ within $5^{\prime \prime}$ of the afterglow location.

(A color version of this figure is available in the online journal.)

telescope 3.7 months after the burst. Images were taken with the BUSCA instrument in the $u^{\prime}, g^{\prime}, r^{\prime}$, and $i^{\prime}$ bands under good seeing condition, with image resolution of $\sim 00^{\prime \prime} 9$. Co-adding was applied to a set of individual images in order to obtain a deeper limiting magnitude. Figure 8 shows one of the images taken in the $r^{\prime}$ band. The center of the circle indicates the afterglow location.

No clear source was detected near the afterglow location within a radius of $5^{\prime \prime}$. The typical $3 \sigma$ upper limits (AB magnitudes) are $u^{\prime} \sim 24.1, g^{\prime} \sim 24.4, r^{\prime} \sim 24.8$, and $i^{\prime} \sim 25.2$. A non-detection of the GRB host galaxy at $r^{\prime} \sim 24.8$ is not surprising since a lot of GRB host galaxies are faint (e.g., Savaglio et al. 2009) or not detected at all (e.g., Ovaldsen et al. 2007). It is also superseded by the much deeper observation reported by Cucchiara et al. (2011) down to an upper limit of $r^{\prime} \sim$ 27.21 mag. At redshift $z=2.22$, a magnitude of $r^{\prime} \sim 27.2$ corresponds to an absolute magnitude $M_{r^{\prime}} \sim-19.1$; the upper limit is fainter than $70 \%$ of the GRB host galaxies compared with large host galaxy samples searched systematically by some groups (see Figure 3 in Jakobsson \& Fynbo 2010; Pozanenko et al. 2008).

\section{THEORETICAL MODELING}

The high-quality broadband data of GRB 110205A allow us to model both prompt emission and afterglow within the framework of the standard fireball shock model and derive a set of parameters that are often poorly constrained from other GRB 
Table 7

Spectral Indices from Synchrotron Spectrum Prediction

\begin{tabular}{lcccc}
\hline \hline Fast cooling & $v<v_{a}$ & $v_{a}<v<v_{c}$ & $v_{c}<v<v_{m}$ & $v_{m}<v$ \\
& 2 & $1 / 3$ & $-1 / 2$ & $-p / 2$ \\
Slow cooling & & & & \\
\hline & $v<v_{a}$ & $v_{a}<v<v_{m}$ & $v_{m}<v<v_{c}$ & $v_{c}<v$ \\
& 2 & $1 / 3$ & $-(p-1) / 2$ & $-p / 2$ \\
\hline Observed mean $^{\mathrm{a}}$ & \multicolumn{5}{c}{} \\
\hline
\end{tabular}

Note. ${ }^{\text {a Spectral index }}=\Gamma+1$, i.e., $\alpha$ and $\beta$ in bandcut model +1 .

observations. In the following, we discuss the prompt emission and afterglow modeling in turn.

\subsection{Prompt Emission Modeling}

\subsubsection{General Consideration}

The mechanism of GRB prompt emission is poorly known. It depends on the unknown composition of the jet, which affects the energy dissipation, particle acceleration, and radiation mechanisms (Zhang 2011). In general, GRB emission can be due to synchrotron, SSC in the regions where kinetic or magnetic energies are dissipated, or Compton scattering of thermal photons from the photosphere. Within the framework of the synchrotron-dominated model (e.g., the internal shock model; Rees \& Mészáros 1994; Daigne \& Mochkovitch 1998, or the internal magnetic dissipation model, e.g., Zhang \& Yan 2011), one can have a broken power-law spectrum. Two cases may be considered according to the relative location of the cooling frequency $v_{c}$ and the synchrotron injection frequency $v_{m}$ : fast cooling $\left(v_{c}<v_{m}\right)$ or slow cooling phase $\left(v_{m}<v_{c}\right)$. The spectral indices are summarized in Table 7 (e.g., Sari et al. 1998).

For GRB 110205A, one may connect the two observed spectral breaks $\left(E_{0}\right.$ and $\left.E_{1}\right)$ to $v_{c}$ and $v_{m}$ in the synchrotron model. Since the spectral index above $E_{1}$ is not well constrained from the data, we focus on the regime below $E_{1}$. The expected spectral density $\left(F_{v}\right)$ power-law index is -0.5 or $-(p-1) / 2$, respectively, for the fast and slow cooling cases. The observed photon index $\beta \sim-1.5$ matches the fast cooling prediction closely. It is also consistent with slow cooling if the electron spectral index $p=2$. For standard parameters, the prompt emission spectrum is expected to be in the fast cooling regime (Ghisellini et al. 2000). Slow cooling may be considered if downstream magnetic fields decay rapidly (Pe'er \& Zhang 2006). The data are consistent with either possibility, with the fast cooling case favored by the close match between the predicted value and the data.

Below $E_{0}$ (which corresponds to $v_{c}$ for fast cooling or $v_{m}$ for slow cooling), the synchrotron emission model predicts a spectral index of $1 / 3$. The observed mean value is $\sim 0.60$, which is harder than the predicted value. Considering the large errors of the spectral indices, this is not inconsistent with the synchrotron model. Furthermore, if the magnetic fields are highly tangled with small coherence lengths, the emission may be in the "jitter" regime. The expected spectral index can then be in the range of 0-1, consistent with the data (Medvedev 2006).

Overall, we conclude that the observed prompt spectrum is roughly consistent with the synchrotron emission model in the fast cooling regime. This is the first time that a clear twobreak spectrum is identified in the prompt GRB spectrum that is roughly consistent with the prediction of the standard GRB synchrotron emission model.

The detection of bright prompt optical emission in GRB 110205A provides new clues to GRB prompt emission physics. The optical flux density of GRB 110205A is $\sim 20$ times above the extrapolation from the best-fit $X / \gamma$-ray spectra. On the other hand, the optical light curve roughly traces that of $\gamma$-rays. This suggests that the optical emission is related to high-energy emission but is powered by a different radiation mechanism or originates from a different emission location. The case is similar to that of GRB 080319B (Racusin et al. 2008) but differs from that of GRB 990123 (Akerlof et al. 1999), where the optical light curve peaks after the main episodes of $\gamma$-ray emission and is likely powered by the RS (Sari \& Piran 1999; Mészáros \& Rees 1999; Zhang et al. 2003; Corsi et al. 2005).

In the following, we discuss several possible interpretations of this behavior, i.e., the synchrotron + SSC model (Kumar \& Panaitescu 2008; Racusin et al. 2008); the internal reverse + forward shock model ( $Y u$ et al. 2009); the two-zone models (Li \& Waxman 2008; Fan et al. 2009); and the dissipative photosphere models (e.g., Pe'er \& Waxman 2005; Pe'er et al. 2006; Giannios 2008; Lazzati et al. 2009, 2011; Lazzati \& Begelman 2010; Toma et al. 2011; Beloborodov 2010; Vurm et al. 2011). We conclude that the synchrotron + SSC model and the photosphere model are disfavored by the data while the other two models are viable interpretations.

\subsubsection{Synchrotron + SSC}

Since the spectral shape of the SSC component is similar to the synchrotron component (Sari \& Esin 2001), the observed two-break spectrum can be in principle due to SSC, while the optical emission is due to synchrotron. This scenario is, however, disfavored since it demands an unreasonably high energy budget. The arguments are the following:

We take interval 2 as an example since its flux varies relatively slowly. Let $h v_{\text {opt }} \sim 10^{-2.3} \mathrm{keV}$ and $h v_{\gamma, p}=E_{0} \sim 10^{0.7} \mathrm{keV}$, the latter being the peak frequency of $F_{v}$ for the SSC component. Observations suggest that $F_{v_{\gamma, p}} / F_{v, \text { opt }} \sim 20$ (see Figure 4 and Table 3). Define $v_{p \text {,syn }}$ as the synchrotron $F_{v}$ peak frequency and $\beta_{\text {opt }}$ as the spectral index around $v_{\text {opt }}\left(F_{v} \propto v^{\beta_{\text {opt }}}\right)$. Then, the Compton parameter can be written as

$$
Y=v_{\gamma, p} F_{v_{\gamma, p}} /\left(v_{p, \text { syn }} F_{v_{p, \text { syn }}}\right) \approx 10^{4.3}\left(v_{p, \text { syn }} / v_{\text {opt }}\right)^{-1-\beta_{\mathrm{opt}}} .
$$

The inverse-Compton (IC) scattering optical depth is $\tau_{e} \sim$ $F_{v_{\gamma, p}} / F_{v_{p, \text { syn }}} \sim Y v_{p, \text { syn }} / v_{\gamma, p} \sim 10^{1.3}\left(v_{p, \text { syn }} / v_{\text {opt }}\right)^{-\beta_{\text {opt }}}$.

One constraint ought to be imposed for the SSC scenario, that is, the high-energy spectrum of the synchrotron component at the lower bound of the X-ray band, i.e., $v_{X}=0.3 \mathrm{keV}$, must be below the observed flux density there. Since the spectral indices below and above $v_{\gamma, p}$ are consistent with $1 / 3$ and $-1 / 2$, respectively, and the synchrotron spectral slope above its peak resembles that of its SSC component, one can express this constraint in terms of $F_{v_{p, \text { syn }}}\left(v_{X} / v_{p, \text { syn }}\right)^{-1 / 2}<F_{v_{\gamma, p}}\left(v_{X} / v_{\gamma, p}\right)^{1 / 3}$. With numbers plugged in, this translates to a lower limit on the Compton parameter:

$$
Y>10^{2.5}\left(v_{p, \text { syn }} / v_{\text {opt }}\right)^{-1 / 2}
$$

The inferred high $Y$ (Equation (5)) and $\tau_{e}$ values would inevitably lead to an additional spectral component due to the second-order IC scattering (Kobayashi et al. 2007; Piran et al. 2009). The second IC $F_{v}$ spectrum peaks at $h v_{\mathrm{ic}, 2} \sim$ 
$h v_{\gamma, p} Y / \tau_{e} \sim 10^{0.7}\left(v_{p, \text { syn }} / v_{\text {opt }}\right)^{-1} \mathrm{MeV}$, with a flux density $\sim \tau_{e} F_{v_{\gamma, p}} \sim 10^{1.3} F_{v_{\gamma, p}}\left(v_{p, \text { syn }} / v_{\mathrm{opt}}\right)^{-\beta_{\mathrm{opt}}}$. The nice fit of the bandcut model to the XRT-BAT-WAM spectrum rules out a second IC peak below $1 \mathrm{MeV}$ (see Figure 4), which poses a constraint $v_{p \text {,syn }} / v_{\text {opt }}<5$.

We then get a constraint $Y \gtrsim 10^{4}$ and $\tau_{e} \gtrsim 10$ ! This would lead to a serious energy crisis due to the second IC scattering. For $v_{p \text {,syn }} / v_{\text {opt }} \ll 1$, the second IC scattering might be in the Klein-Nishina regime and then be significantly suppressed, but the synchrotron peak flux density would be self-absorbed, causing the seed flux to be insufficient for the first IC scattering (Piran et al. 2009). In conclusion, the SSC scenario is ruled out due to the high $Y$ value inferred.

\subsubsection{Internal Reverse-Forward Shocks}

Next we consider the internal shock model by calculating synchrotron emission from the RS and FS due to the collision of two discrete cold shells (e.g., Rees \& Mészáros 1994; Daigne \& Mochkovitch 1998; Yu et al. 2009). If the two shells have high density contrast, the synchrotron frequencies would peak around the $\gamma$-ray (reverse) and optical (forward) bands, respectively.

We first derive the frequency and flux ratio between the two shocks (Kumar \& McMahon 2008). We define shell " 1 " as the fast-moving, trailing shell and shell " 2 " as the slower, leading shell. We use subscript " $s$ " to represent the shocked region. The pressure balance at the contact discontinuity gives (e.g., Shen et al. 2010)

$$
\left(\Gamma_{1 s}^{2}-1\right) n_{1}=\left(\Gamma_{2 s}^{2}-1\right) n_{2},
$$

where $\Gamma_{1 s}$ and $\Gamma_{2 s}$ are the Lorentz factors (LFs) of the unshocked shells, respectively, measured in the shocked region rest frame, and $n_{1}, n_{2}$ are the unshocked shell densities measured in their own rest frames, respectively. This equation is exact and is valid for both relativistic and sub-relativistic shocks. Using Lorentz transformation of LFs, the above equation can give the shocked region LF $\Gamma_{s}$ for given $n_{1} / n_{2}, \Gamma_{1}$, and $\Gamma_{2}$.

We assume that $\epsilon_{e}, \epsilon_{B}$, and $p$ are the same for both RS and FS. In the shocked region, the magnetic field energy density is $U_{B}^{\prime}=B^{\prime 2} / 8 \pi=4 \bar{\Gamma}(\bar{\Gamma}-1) \epsilon_{B} n$, where $\bar{\Gamma}$ is the relative LF between the downstream and upstream of the shock, which corresponds to $\Gamma_{1 s}$ and $\Gamma_{2 s}$ for RS and FS, respectively. Since the internal energy density is the same in the RS and FS regions (due to pressure balance at contact discontinuity), it is obvious that the two shocked regions have the same $B^{\prime}$, independent of the strengths of the two shocks. The injection energy and the cooling energy of the electrons are $\gamma_{m} \propto(\bar{\Gamma}-1)$ and $\gamma_{c} \propto 1 /\left(U_{B}^{\prime} t^{\prime}\right)$, respectively. Since synchrotron frequency $v \propto \Gamma_{s} B^{\prime} \gamma^{2}$, one finds the frequency ratios to be

$$
\begin{gathered}
\frac{v_{m, 1}}{v_{m, 2}}=\frac{\left(\Gamma_{1 s}-1\right)^{2}}{\left(\Gamma_{2 s}-1\right)^{2}}, \\
\frac{v_{c, 1}}{v_{c, 2}}=1 .
\end{gathered}
$$

Thus, the injection frequency ratio can be determined for given $\Gamma_{1}, \Gamma_{2}$, and shell density ratio $n_{1} / n_{2}$. We numerically calculate $v_{m, 1} / v_{m, 2}$ as a function of $n_{1} / n_{2}$ and plot it in Figure 9, and we find that for different shell LF ratios $v_{m, 1} / v_{m, 2}$ lies between $\left(n_{1} / n_{2}\right)^{-1}$ and $\left(n_{1} / n_{2}\right)^{-2}$.

The maximum flux density is $F_{v, \max } \propto \Gamma_{s} N_{e} B^{\prime}$, where $N_{e}$ is the total number of the shocked electrons. So one has $F_{v, \max , 1} / F_{v, \max , 2}=m_{1} / m_{2}$, where $m$ is the shock swept-up mass. We calculate the mass ratio in the rest frame of the shocked region. In this frame the density of the shocked fluid is $4 \bar{\Gamma} n$; the rate of mass sweeping is proportional to the sum of the shock front speed and the unshocked fluid speed. We then get

$$
\frac{m_{1}}{m_{2}}=\frac{\Gamma_{1 s} n_{1}\left(\beta_{r s, s}+\beta_{1 s}\right)}{\Gamma_{2 s} n_{2}\left(\beta_{s f, s}+\beta_{2 s}\right)},
$$

where the speeds $\beta$ are all defined positive and are measured in the shocked fluid rest frame; the subscripts " $r$ " and " $f s$ " refer to the RS and FS front, respectively. From the shock jump conditions (e.g., Blandford \& McKee 1976), one gets

$$
\beta_{r s, s}=\frac{(\hat{\gamma}-1) \beta_{1 s}}{1+1 / \Gamma_{1 s}},
$$

where $\hat{\gamma}$ is the adiabatic index for a relativistic fluid. Using an empirical relation $\hat{\gamma}=(4 \bar{\Gamma}+1) /(3 \bar{\Gamma})$ to smoothly connect the sub-relativistic shock regime to the relativistic shock regime, we obtain $\left(\beta_{r s, s}+\beta_{1 s}\right)=4 \beta_{1 s} / 3$. A similar result applies to the FS front. Thus, Equation (10) becomes

$$
\frac{F_{v, \max , 1}}{F_{v, \max , 2}}=\frac{m_{1}}{m_{2}}=\frac{\Gamma_{1 s} \beta_{1 s} n_{1}}{\Gamma_{2 s} \beta_{2 s} n_{2}}=\left(\frac{n_{1}}{n_{2}}\right)^{1 / 2},
$$

where we have used Equation (7). This result is also numerically plotted in Figure 9.

According to Figure 9, in the internal RS-FS model, the optical emission shell must have a higher pre-shock density and a larger shock swept-up mass and hence should have a higher $F_{v, \max }$. The analysis of GRB 110205A prompt X/ $\gamma$-ray spectrum suggests that the characteristic synchrotron frequencies are $h v_{c, \gamma} \sim 5 \mathrm{keV}$ and $h v_{m, \gamma} \sim 300 \mathrm{keV}$ (see Section 3.1); then we have $F_{\nu, \max , \gamma} / F_{\nu, \mathrm{opt}} \sim 20$ and $v_{m, \gamma} / v_{\mathrm{opt}} \sim$ $6 \times 10^{4}$. Therefore, if the internal RS-FS model would work for this burst, the maximum flux density of the optical producing shell $F_{v \text {, max }}$ must be $\gg F_{v \text {,opt }}$; since the cooling frequencies of the two shells are equal (Equation (9)), the optical shell must be in the slow cooling $\left(v_{m}<v_{c}\right)$ regime.

In order to have the observed $F_{v \text {,opt }}$ much smaller than the optical shell $F_{v \text {,max }}$, either $v_{\text {opt }}$ has to be far below or far above $v_{m}$, or the self-absorption frequency has to be $v_{a}>v_{\text {opt }}$, or both. In the following, we use the observed $F_{v, \max , \gamma} / F_{v, \text { opt }}$ and $v_{m, \gamma} / v_{\text {opt }}$ as constraints and $v_{a} / v_{\text {opt }}$ (for the optical shell) as a free parameter and derive the permitted relation between $v_{m, \gamma} / v_{m \text {,opt }}$ and $n_{\gamma} / n_{\text {opt }}$, where $n_{\gamma} / n_{\text {opt }}$ is the density ratio of the $\gamma$-ray shell over the optical shell and is given by the maximum flux density ratio of the two shells (Equation (12)). We then overlay the permitted relations onto the internal RS-FS model predictions shown in Figure 9, right panel, in order to find the permitted model parameter values, i.e., shell LF ratio $\Gamma_{\text {fast }} / \Gamma_{\text {slow }}$, shell density ratio $n_{\gamma} / n_{\mathrm{opt}}$, and $v_{a} / v_{\mathrm{opt}}$.

For the optical shell, the relation between $F_{v \text {,opt }}$ and $F_{v \text {, max }}$ is determined according to the standard broken power-law synchrotron spectrum (e.g., Sari et al. 1998; Granot \& Sari 2002), depending on the free parameter $v_{a} / v_{\text {opt }}$, which varies from $<1$ to $>1$. In addition, we impose an additional constraint that the high-energy spectrum of the optical producing shell emission should not exceed the observed flux density at the lower bound of the X-ray band, i.e., at $v_{X}=0.3 \mathrm{keV}$, so that the spectral slope there would not be inconsistent with the observed one. The final results are shown in Figure 10.

From Figure 10, we conclude that the prompt SED data of GRB 110205A can be reproduced by the internal RS-FS synchrotron model under the following conditions: $v_{m, \gamma} / v_{m, \text { opt }} \approx$ 

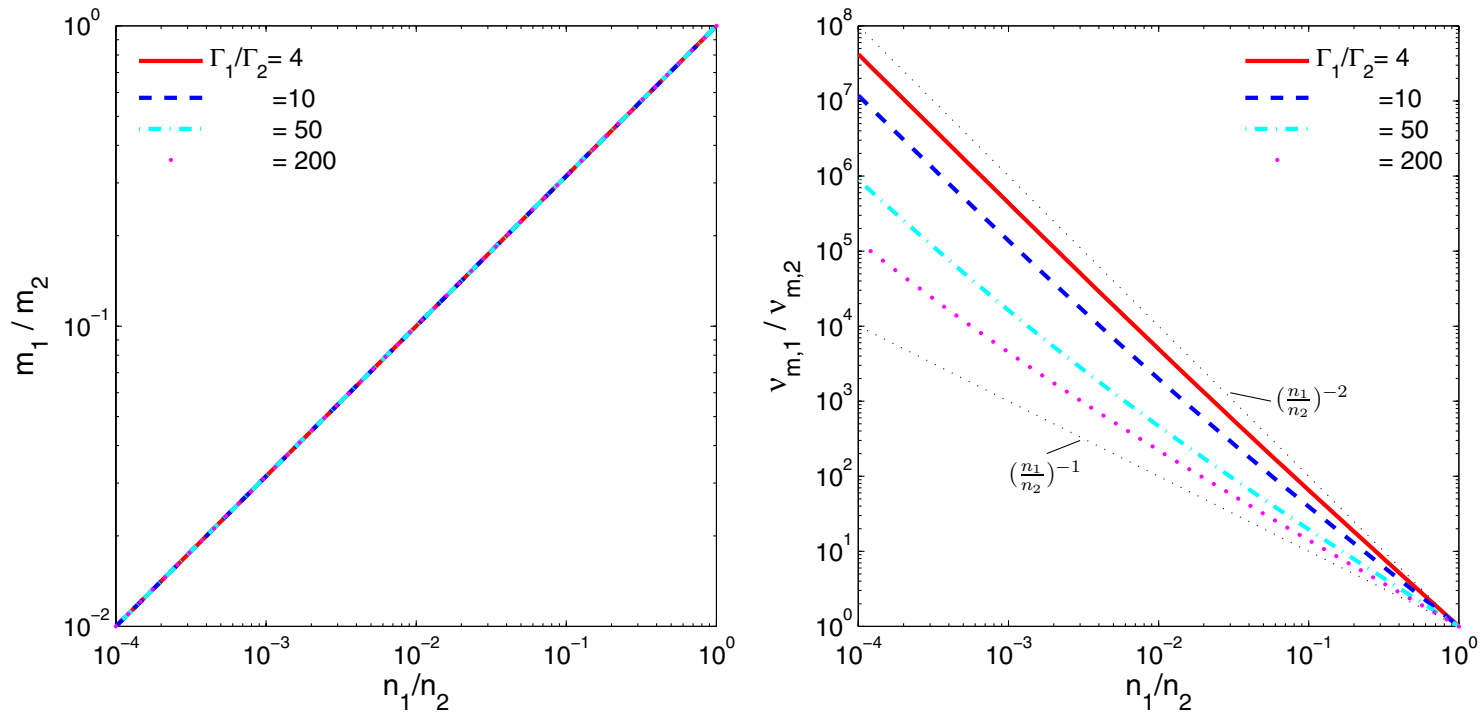

Figure 9. Ratios of the shock swept-up masses and synchrotron injection frequencies between the pair of shocks due to collision of two cold relativistic shells. The results are valid for both sub-relativistic and relativistic shocks and are insensitive to $\Gamma_{2}$ as long as $\Gamma_{2} \gg 1$. Note that these results can be extrapolated to the region of $n_{1}>n_{2}$, and each result is symmetric about the $(1,1)$ point.

(A color version of this figure is available in the online journal.)

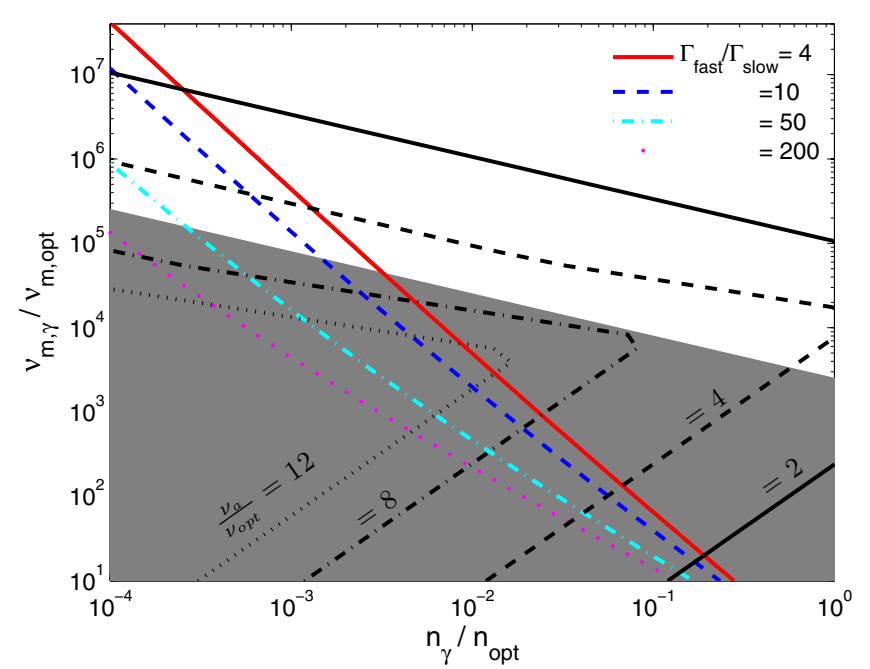

Figure 10. GRB 110205A-permitted $v_{m, \gamma} / v_{m}$ opt vs. $n_{\gamma} / n_{\text {opt }}$ relations for varying $v_{a} / v_{\text {opt }}$ values (black lines), superimposed on the internal RS-FS model predictions (colored lines, from the right panel of Figure 9). The locations where the data-permitted and the model-predicted relations intersect correspond to the specific model parameter values with which the model could work for GRB 110205A. Electron index $p=3$ is assumed. The shaded region is forbidden because there the flux density at $v_{X}=0.3 \mathrm{keV}$ in the emission spectrum of the optical producing shell will exceed the observed value of $F_{v, X}$, causing the spectral slope to be inconsistent with the observed one. Note that for $v_{a}<v_{\text {opt }}$ the data-permitted $v_{m, \gamma} / v_{m, \text { opt }}$ vs. $n_{\gamma} / n_{\text {opt }}$ relations have no intersection with the internal RS-FS predictions unless $n_{\gamma} / n_{\text {opt }}$ is unreasonably small.

(A color version of this figure is available in the online journal.)

$10^{6}-10^{7}, n_{\gamma} / n_{\mathrm{opt}} \approx 10^{-4}-10^{-3}$, and $v_{a} / v_{\mathrm{opt}} \approx 2-6$ for the optical shell; and the LF ratio between the two shells falls into a wide range $\sim 4-100$. In Figure 10, the electron index $p=3$ has been adopted. A smaller $p$ value only increases the inferred $v_{m, \gamma} / v_{m \text {,opt }}$ and decreases $n_{\gamma} / n_{\text {opt }}$ both by a factor $<10$, without affecting the conclusion.

\subsubsection{Emission Radius}

The distance of the GRB emission region from the central engine $\left(R_{\mathrm{GRB}}\right)$ has been poorly constrained. If prompt opti- cal data are observed, one may apply the constraint on the synchrotron self-absorption frequency $\left(v_{a}\right)$ to constrain $R_{\mathrm{GRB}}$ (Shen \& Zhang 2009). One needs to assume that the optical and $\gamma$-ray emissions are from essentially the same radius in order to pose such a constraint. This is the simplest scenario and is valid for some scenarios we have discussed, e.g., the internal RS-FS model as discussed in Section 4.1.3. In the following, we derive the emission radius based on the one-zone assumption, bearing in mind that optical and gamma-ray emissions can come from different zones.

For GRB 110205A, the synchrotron optical emission from the optical producing shell is self-absorbed and has the following frequency ordering: $v_{m}<v_{\text {opt }}<v_{a}<v_{c}$ (Section 4.1.3). For such a frequency ordering, $v_{a}$ is determined by (Shen $\&$ Zhang 2009)

$$
2 \gamma_{a} m_{e} v_{a}^{2}=F_{v_{a}}\left(\frac{D_{L}}{R}\right)^{2} \frac{(1+z)^{3}}{\Gamma},
$$

where $D_{L}$ is the luminosity distance, $F_{v_{a}}$ is the observed flux density at $v_{a}$, and $\gamma_{a}=\left[16 m_{e} c(1+z) v_{a} /\left(3 e B^{\prime} \Gamma_{s}\right)\right]^{1 / 2}$ is the LF of electrons whose synchrotron frequency is $v_{a}$. Expressing $F_{v_{a}}$ in terms of the self-absorbed optical flux density, $F_{v_{a}}=F_{v, \text { opt }}\left(v_{a} / v_{\text {opt }}\right)^{5 / 2}$, we find that $v_{a}$ is canceled out in the above equation:

$$
2 m_{e}\left(\frac{16 m_{e} c}{3 e B^{\prime}}\right)^{1 / 2}\left(\frac{R}{D_{L}}\right)^{2} \frac{(1+z)^{7 / 2}}{\Gamma_{s}^{3 / 2}}=F_{v, \mathrm{opt}} v_{\mathrm{opt}}^{-5 / 2}
$$

For an observed average $F_{v, \text { opt }} \approx 10^{1.8} \mu \mathrm{Jy}, h v_{\text {opt }} \approx 10^{-2.3} \mathrm{keV}$, and other numbers for GRB 110205A, we obtain

$$
R_{\mathrm{GRB}} \approx 2.8 \times 10^{13}\left(\frac{\Gamma_{s}}{250}\right)^{3 / 4}\left(\frac{B^{\prime}}{10^{5} \mathrm{G}}\right)^{1 / 4} \mathrm{~cm} .
$$

We have normalized $\Gamma_{s}$ to 250 and $B^{\prime}$ to $10^{5} \mathrm{G}$. The former normalization can be justified from the afterglow data (Section 4.2.1). The value of $B^{\prime}$ is loosely determined and may be estimated by $B^{\prime} \sim 10^{6} \zeta L_{52} R_{13} \Gamma_{2} \mathrm{G}$, where $\zeta \leqslant 1$ is a constant parameter (Zhang \& Mészáros 2002). This gives 
$B^{\prime} \sim 10^{5} \mathrm{G}$ for the parameters of GRB 110205A. Interpreting $E_{1}$ as $v_{m}$ would also give rise to $B^{\prime} \sim 10^{5} \mathrm{G}$ if $\Gamma_{1 s} \sim 3$ and $\epsilon_{e} \sim 0.1$. We note that $R_{\mathrm{GRB}}$ is a weak function of $B^{\prime}$, so that an estimate $R_{\mathrm{GRB}} \approx 3 \times 10^{13} \mathrm{~cm}$ is robust. This radius is consistent with the expectation of the internal shock model (e.g., Piran 2005).

Note that in the Shen \& Zhang (2009) method one has to assume that both the optical and $\gamma$-rays belong to the same continuum component and partly rely on the $\gamma$-ray lowenergy spectral slope to constrain the $v_{a}$ location. However, this assumption is discarded in the case of GRB 110205A: it is inferred that in the internal RS-FS model the optical shell has $v_{\mathrm{opt}}<v_{a}$ (Section 4.1.3), for which $R_{\mathrm{GRB}}$ is worked out without the aid of the $\gamma$-ray spectral information.

\subsubsection{Two-zone Models}

The GRB 110205A prompt observations might be also interpreted if the optical emission region is decoupled from the $\gamma$-ray/X-ray emission regions. There are models that envisage that the $\gamma$-rays are produced in internal shocks at small radii between shells with large LF contrasts, while the optical emission is generated in internal shocks at larger radii by shells with lower magnetic fields and smaller LF contrasts. This can happen in two scenarios. According to Li \& Waxman (2008), even after collisions and mergers of shells with large LF contrasts, the outflow still comprises discrete shells with variations, though with reduced relative LFs, which could lead to the "residual" collisions at larger radii. Fan et al. (2009) considered a neutron-rich GRB outflow in which free neutrons are decoupled from the proton shells until decaying at large radii. Violent collisions among the proton shells occur at smaller radii, while some later-ejected, slower, proton shells catch up with the decayed neutron shells at large radii and give weaker collisions. Both scenarios might work for GRB 110205A, since in either case, the large-radii collisions would bear a similar temporal information as the small-radii collisions, rendering a coarse optical- $\gamma$-ray correlation. A defining property of these two-zone scenarios is that the optical pulses should display a larger variability timescale $\delta t$ than the $\gamma$-ray pulses, and they should lag behind the $\gamma$-ray pulses by $\sim R_{\mathrm{opt}} / 2 \Gamma^{2} \mathrm{c} \sim$ $0.2 \mathrm{~s} R_{\mathrm{opt}, 15} \Gamma_{2.5}^{-2} \mathrm{~s}$. These predictions could be tested by future, high temporal resolution, prompt optical observations of similar bursts.

\subsubsection{Dissipative Photosphere Emission Model}

Recently, several independent groups (e.g., Giannios 2008; Toma et al. 2011; Beloborodov 2010; Lazzati \& Begelman 2010; Vurm et al. 2011) have developed an improved version of the photosphere emission model of GRB prompt emission. This model invokes energy dissipation in the Thomson-thin layer of the photosphere, so that the photosphere spectrum deviates from the thermal form through IC upscattering. The same electrons also emit synchrotron photons, which may account for the optical excess. A difficulty of this scenario is that the low-energy spectral index below $E_{p}$ is too hard (e.g., $\alpha=+0.4$; Beloborodov $2010)$ to account for the observations $(\alpha=-1.5)$. The data of GRB 110205A (double breaks and spectral slopes) of the $\mathrm{X} / \gamma$-ray component do not comply with the predictions of this model but are rather consistent with the standard synchrotron model (see Section 4.1.1). We conclude that the dissipative photosphere model does not apply at least to this burst.

\subsection{Afterglow Modeling}

\subsubsection{Initial Lorentz Factor}

For both Scenarios I and II, the optical peak time $t_{p}=1045 \pm$ $63 \mathrm{~s}$ corresponds to the deceleration time. Since this time is much longer than $T_{90}(\sim 257 \mathrm{~s})$, it is pertinent to consider a "thin" shell regime (Sari \& Piran 1995). The peak time can then be used to estimate the initial Lorentz factor of the ejecta (e.g., Mészáros 2006; Molinari et al. 2007): $\Gamma_{0} \sim 560\left(E_{\gamma, \text { iso }, 52} / \eta_{0.2} n_{0} t_{p, z, 1}^{3}\right)^{1 / 8}$, where $E_{\gamma, \text { iso,52 }}$ is the isotropic equivalent energy in units of $10^{52} \mathrm{erg} \mathrm{s}^{-1}, \eta_{0.2}$ is the radiative efficiency in units of $0.2, n_{0}$ is local density in units of $\mathrm{cm}^{-3}$, and $t_{p, z, 1}$ is the peak time corrected for cosmological time dilation in units of $10 \mathrm{~s}$. For GRB 110205A, with redshift $z=2.22$ and fluence of $2.7_{-0.4}^{+0.7} \times$ $10^{-5} \mathrm{erg} \mathrm{cm}^{-2}$ (15-3000 keV; Sakamoto et al. 2011c), we derive the rest-frame $1 \mathrm{keV}-10 \mathrm{MeV}$ isotropic energy $E_{\gamma \text {,iso }}=46_{-7}^{+4} \times$ $10^{52} \mathrm{erg}$. With $t_{p, z, 1}=32.4 \pm 1.8$, we finally estimate

$$
\Gamma_{0}=245_{-10}^{+7}\left(\eta_{0.2} n_{0}\right)^{-1 / 8} .
$$

This value follows the empirical relation $\Gamma_{0} \sim 182 \mathrm{E}_{\gamma, \text { iso, } 52}^{0.25 \pm 0.03}$ recently found by Liang et al. (2010) within the $2 \sigma$ range.

\subsubsection{Light Curves}

\section{Scenario I}

In this scenario, the optical bump around $t_{p}$ is mostly contributed by the FS. Fitting the light curves, one can constrain the temporal slopes of the RS component. In the $R$ band, the temporal indices are $\alpha_{r 1}=3.32 \pm 1.2$ (rising phase) and $\alpha_{r 2}=$ $-5.90 \pm 1.0$ (decaying phase), while in the X-ray band, they are $\alpha_{r 1}=5.19 \pm 1.3$ (rising) and $\alpha_{r 2}=-8.26 \pm 1.3$ (decaying). The steep rising slope $(\sim 4)$ is consistent with the expectation in the thin shell ISM RS model (e.g., Kobayashi 2000; Zhang et al. 2003). The decaying slopes look too steep as compared with the theoretically expected values (e.g., roughly $-2-\beta$ for the socalled curvature effect; Kumar \& Panaitescu 2000). However, strictly speaking, the expected decay index is valid when the time zero point is placed to $t_{p}$. The results are therefore not inconsistent with the theoretical expectations. Compared with other GRBs with the RS emission identified (which typically peaks around or shortly after the $T_{90}$ duration), an $\mathrm{RS}$ emission peaking at $\sim 1100 \mathrm{~s}$ after the burst is rare and has not been seen before (though previously, the optical brightening in the ultra-long GRB 091024 was claimed by Gruber et al. 2011 to be caused by the rising RS, but its data coverage is very sparse and the RS origin was not exclusively determined, e.g., it could also be due to the FS peak in a wind medium).

For the FS component, the rising index is set to +3 during the fitting for both the optical and X-ray bands. The decay index after the peak is fitted to $\alpha_{o f 2}=-1.50 \pm 0.04$ in the optical band and $\alpha_{X f 2}=-1.54 \pm 0.1$ in the X-ray band. We also constructed two SEDs at the epochs $5.9 \mathrm{ks}$ and $35 \mathrm{ks}$ during the decay phase. We find that the only model that satisfies the closure relation (e.g., Sari et al. 1998; Granot \& Sari 2002; Zhang \& Mészáros 2004) is the ISM model in the $v_{m}<v_{o}<v_{x}<v_{c}$ spectral regime. For example, our SED at $5.9 \mathrm{ks}$ gives a spectral slope $\beta \sim-1.01 \pm 0.02$ across the entire energy band. The optical temporal decay index $\alpha_{o}=-1.50 \pm 0.04$ matches the expected closure relation $\alpha=(3 / 2) \beta$ well. The X-ray decay slope $\alpha_{X f 2}=-1.54 \pm 0.1$ within $1 \sigma$ error is consistent with the closure relation. The electron energy index, $p=-2 \beta+1 \sim 3.0$, is also consistent with its value derived from the temporal 
index $p=(-4 \alpha+3) / 3=3.0 \pm 0.08$ derived from $\alpha_{o}$ and $p=3.05 \pm 0.14$ derived from $\alpha_{x}$.

At late times around $\sim 10^{5} \mathrm{~s}$, the decay index becomes steeper in both the optical and X-ray bands, which is probably caused by a jet break. The simultaneous fit suggests a $t_{j b}=$ $1.0 \pm 0.2 \times 10^{5} \mathrm{~s}$ from the two band light curves. The post-break temporal indices are consistent with a jet break without significant sideways expansion, which is predicted to be steeper by $3 / 4$. But the conclusion is not conclusive due to large errors. The jet angle can be calculated using (Sari et al. 1999) $\theta=$ $1 / 6\left(t_{j b} / 1+z\right)^{3 / 8}\left(n \eta_{0.2} / E_{\gamma, \text { iso }, 52}\right)^{1 / 8}$. Taking $t_{j b} \simeq 1.0 \times 10^{5} \mathrm{~s}=$ 1.2 days and $E_{\gamma, \text { iso }, 52}=46.0$, we derive $\theta=\left(4.1_{-1.0}^{+3.5}\right)^{\circ}$. With a beaming factor of $\theta^{2} / 2$, the corresponding jet-angle-corrected energy is $E_{\gamma}=1.2 \times 10^{51} \mathrm{erg}$.

\section{Scenario II}

In this scenario, the early steep rise and bright peak are dominated by the RS component only. It can also be well explained by an ISM model in the thin shell regime. Within this scenario, the FS component shows up and peaks later. The FS peak is defined by $v_{m}$ crossing the optical band. There should also be a break time in the FS light curve at the RS peak $t_{p}$, which is caused by the onset of the afterglow. After the FS peak, the afterglow analysis is similar to Scenario I. We find that the best afterglow model for this scenario is the ISM model assuming $v_{m}<v_{c}<v_{o}<v_{x}$. From the spectral index $\beta=-1.01$, one can derive $p=2.02$. Since $\alpha \sim 1.0$ for both optical and X-ray, the closure relation $\alpha=(3 \beta+1) / 2$ is well satisfied.

The X-ray bump around $1000 \mathrm{~s}$ is also consistent with being the emission from the RS. Our SED fit (see Table 6) near the peak at $1.13 \mathrm{ks}$ shows that the best fits are a single power-law (LMC and SMC model) or a broken power-law model (LMC model) with the break energy $\sim 2.9 \mathrm{keV}$. The result suggests that the optical and X-ray bands belong to the same emission component with $v_{m}<v_{\text {opt }}<v_{X}<v_{c}$ (for single power law) or $v_{m}<v_{\text {opt }}<v_{c}<v_{X}$ (for broken power law). In the case of a thin ejecta shell and ISM, the synchrotron cooling frequency at the time when the RS crosses the ejecta, $t_{p}$, can be estimated to be $v_{c}\left(t_{p}\right)=2 \times 10^{26} \Gamma^{-4}\left(\epsilon_{B} n\right)^{-3 / 2}(1+z) t_{p}^{-2} \mathrm{~Hz}$ (also see Kobayashi 2000). Adopting $\Gamma \sim 250, z=2.22$, and $t_{p}=1.1$ $\mathrm{ks}$, which are relevant for GRB 110205A, this would require $\epsilon_{B} n \sim 10^{-5} \mathrm{~cm}^{-3}$. Such a value, although in the low end of the generally anticipated parameter distribution range, is not impossible.

The jet break time derived from this scenario is somewhat earlier than Scenario I, which is $\sim(5.44 \pm 0.2) \times 10^{4} \mathrm{~s}$ from the simultaneous fitting. Adopting this break time, we derived a jet angle for this scenario of $\theta=\left(3.2_{-0.9}^{+2.6}\right)^{\circ}$, which corresponds to a jet-angle-corrected energy of $E_{\gamma}=7.2 \times 10^{50} \mathrm{erg}$.

\subsubsection{RS Magnetization}

The composition of the GRB ejecta is still not well constrained. Zhang et al. (2003) suggested that bright optical flashes generally require that the RS is more magnetized than the FS, namely, the ejecta should carry a magnetic field flux along with the matter flux (see also Fan et al. 2002; Panaitescu \& Kumar 2004 for case studies). Since GRB 110205A has a bright RS component, it is interesting to constrain the RS parameters to see whether it is also magnetized. For both Scenarios I and II, since the peak time and maximum flux of both FS and RS can be determined (also shown in Table 4), one can work out the constraints on the RS magnetization following the method delineated in Zhang et al. (2003). The same notations are adopted here as in Zhang et al. (2003).

For Scenario I, the FS peaks at $t_{\times}$, where $v_{m, f}<v_{R}<v_{c, f}$, and then decays as $F_{\nu, f} \propto t^{-3\left(p_{f}-1\right) / 4}$. For Scenario II, in the FS, one has $v_{R} \leqslant v_{m, f}<v_{c, f}$ at $t_{\times}$. The FS light curve still rises as $t^{1 / 2}$, until reaching $t_{p, f}$, where $v_{R}=v_{m, f}<v_{c, f}$. We define $\left(t_{p, r}, F_{v, p, r}\right)$ and $\left(t_{p, f}, F_{v, p, f}\right)$ as the peak times and peak flux densities in optical for RS and FS, respectively. Similar to those presented in Zhang et al. (2003), it follows naturally from the above that

$$
\begin{gathered}
\Re_{t} \equiv \frac{t_{p, f}}{t_{p, r}}= \begin{cases}1, & \text { for Scenario I } \\
\Gamma_{\times}^{4 / 3} \Re_{B}^{-2 / 3} \Re_{\nu}^{-2 / 3}, & \text { for Scenario II }\end{cases} \\
\Re_{F} \equiv \frac{F_{\nu, p, r}}{F_{v, p, f}}= \begin{cases}\Gamma_{\times}^{2-p_{f}} \Re_{B}^{\frac{p_{f}+1}{2}} \Re_{\nu}^{\frac{p_{f}-p_{r}}{2}}, & \text { for Scenario I } \\
\Gamma_{\times} \Re_{B} \Re_{\nu}^{-\frac{p_{r}-1}{2}}, & \text { for Scenario II. }\end{cases}
\end{gathered}
$$

Note that Scenario I is actually a special case of $\Re_{t}=1$ of the more general Scenario II. The results are identical to those in Zhang et al. (2003) except that we do not use the RS decay slope $\alpha_{r, 2}$ after $t_{\times}$as one of the parameters. This was to avoid the ambiguity of the blastwave dynamics after the shock crossing. Instead, we keep $p_{r}$ in the formulae and determine $p_{r}$ from the better-understood RS rising slope before $t_{\times}: \alpha_{r, 1}=\left(6 p_{r}-3\right) / 2$.

For Scenario I, one can derive $\Re_{t} \sim 1, p_{r} \approx 2, p_{f} \approx 3$, and $\mathfrak{R}_{F} \approx 0.5$. Note that the entire optical peak around $t_{\times}$ is contributed mainly from the FS, which means $p_{r}$ is poorly constrained. So we take $p_{r}=p_{f} \approx 3$. Then from Equation (18) we get

$$
\Re_{B} \sim 7 \Gamma_{\times, 2}^{1 / 2} \sim 7.7
$$

For Scenario II, $\mathfrak{R}_{t} \approx 3.6, \mathfrak{R}_{F} \approx 16.5$, and $p_{r} \approx 2.3$. Plugging in numbers and from Equation (17), we have $\Re_{B} \Re_{v} \sim$ $\Gamma_{\times}^{2} / 6.8$. Combining it with Equation (18) and canceling out $\Re_{\nu}$, we get

$$
\Re_{B} \sim 7 \Gamma_{\times, 2}^{0.18} \sim 7.2
$$

The numerical values are obtained by taking $\Gamma_{\times}=\Gamma_{0} / 2 \sim 120$.

In both scenarios, the magnetic field strength ratio $\mathfrak{R}_{B} \equiv$ $B_{r} / B_{f}$ is $\sim 7$. This suggests that the RS is more magnetized than the FS. Since the magnetic field in the FS is believed to be induced by plasma instabilities (Weibel 1959; Medvedev \& Loeb 1999; Nishikawa et al. 2009), a stronger magnetic field in the RS region must have a primordial origin, i.e., from a magnetized central engine.

\subsubsection{Discussion}

Comparing with the other recent work on GRB 110205A (Cucchiara et al. 2011; Gao 2011; Gendre et al. 2011), our Scenario II analysis, which concludes that the bright optical peak around $1000 \mathrm{~s}$ is dominated by the RS emission, agrees with that by Gendre et al. (2011) and Gao (2011). Our Scenario I analysis, though close to the conclusion by Cucchiara that it is dominated by the FS emission, has slight difference, as we also consider the RS contribution in our Scenario I.

Both scenarios in our analysis can interpret the general properties of the broadband afterglow. However, each scenario has some caveats. For Scenario I, as explained above, the best-fit model light curve is not steep enough to account for the data in the UVOT- $u$ band ( $\alpha \sim 5$ for $R$ and $\alpha \sim 6$ for UVOT- $u$ if we apply a single broken power-law fitting). Since the inconsistency only occurs in the bluest band with adequate data to constrain 
the rising slope, we speculate that the steeper rising slope may be caused by a decreasing extinction with time near the GRB. No clear evidence of the changing extinction has been observed in other GRBs. In any case, theoretical models have suggested that dust can be destructed by strong GRB X-ray and UV flashes along the line of sight, so that a time-variable extinction is not impossible (e.g., Waxman \& Draine 2000; Fruchter et al. 2001; Lazzati et al. 2002a; De Pasquale et al. 2003). For Scenario II, the model cannot well fit the X-ray peak around $1100 \mathrm{~s}$. The main reason is that the required $\mathrm{RS}$ component to fit the optical light curve is not as narrow as that invoked in Scenario I. It is possible that the X-ray feature is simply an X-ray flare due to late central engine activities, which have been observed in many GRBs (e.g., Burrows et al. 2005b; Liang et al. 2006; Chincarini et al. 2007).

In the late optical light curve around $5 \times 10^{4} \mathrm{~s}$, there is a re-brightening bump observed by LOT in four bands. Such bumps have been seen in many GRBs (e.g., GRB 970508, Galama et al. 1998; GRB 021004, Lazzati et al. 2002b; GRB 050820, Cenko et al. 2006; GRB 071025, Updike et al. 2008; GRB 100219A, Mao et al. 2012), which are likely caused by the medium-density bumps (e.g., Lazzati et al. 2002b; Dai \& Wu 2003; Nakar \& Granot 2007; Kong et al. 2010). Microlensing is another possibility (e.g., Garnavich et al. 2000; Gaudi et al. 2001; Baltz \& Hui 2005), although the event rate is expected to be rather low.

Interestingly, linear polarization at a level of $P \sim 1.4 \%$ was measured by the $2.2 \mathrm{~m}$ telescope at Calar Alto Observatory (Gorosabel et al. 2011) 2.73-4.33 hr after the burst. During this time, the afterglow is totally dominated by the FS in Scenario I or mostly dominated by the FS (with a small contamination from the RS) in Scenario II. The measured linear polarization degree is similar to several other detections in the late afterglow phase (e.g., Covino et al. 1999, 2003; Greiner et al. 2003; Efimov et al. 2003), which is consistent with the theoretical expectation of synchrotron emission in external shocks (e.g., Gruzinov \& Waxman 1999; Sari 1999; Ghisellini \& Lazzati 1999).

\section{SUMMARY}

We have presented a detailed analysis of the bright GRB 110205A, which was detected by both Swift and Suzaku. Thanks to its long duration, Swift XRT, UVOT, ROTSE-IIIb, and BOOTES telescopes were able to observe when the burst was still in the prompt emission phase. Broadband simultaneous observations are available for nearly 200 s, which makes it one of the exceptional opportunities to study the SED during the prompt phase. The broadband time-resolved spectra are well studied. For the first time, an interesting two-break energy spectrum is identified throughout the observed energy range, which is roughly consistent with the synchrotron emission spectrum predicted by the standard GRB internal shock model. Shortly after the prompt emission phase, the optical light curve shows a bump feature around $1100 \mathrm{~s}$ with an unusual steep rise $(\alpha \sim$ $5.5)$ and a bright peak $(R \sim 14.0 \mathrm{mag})$. The X-ray band shows a bump feature around the same time. This is followed by a more normal decay behavior in both optical and X-ray bands. At late times, a further steepening break is visible in both bands.

The rich data in both the prompt emission and afterglow phase make GRB 110205A an ideal burst to study GRB physics, to allow the study of the emission mechanisms of GRB prompt emission and afterglow, and to constrain a set of parameters that are usually difficult to derive from the data. It turns out that the burst can be well interpreted within the standard fireball shock model, making it a "textbook" GRB. We summarize our conclusions as follows:

1. The two-break energy spectrum is highly consistent with the synchrotron emission model in the fast cooling regime. This is consistent with the internal shock model or the magnetic dissipation model that invokes first-order Fermi acceleration of electrons.

2. The prompt optical emission is $\sim 20$ times greater than the extrapolation from the $\mathrm{X} / \gamma$-ray spectrum. Our analysis rules out the synchrotron $+\mathrm{SSC}$ model to interpret the optical $+\mathrm{X} / \gamma$-ray emission. We find that the prompt emission can be explained by a pair of reverse/forward shocks naturally arising from the conventional internal shock model. In a two-shell collision, the synchrotron emission from the slower shock that enters the denser shell produces optical emission and is self-absorbed, while that from the faster shock entering the less dense shell produces the $\mathrm{X} / \gamma$-ray emission. The required density ratio of two shells is $\sim 10^{-4}$ to $10^{-3}$.

3. If the optical and gamma-ray emissions originate from the same radius, as is expected in the internal forward/reverse shock model, one can pinpoint the prompt emission radius to $R \sim 3 \times 10^{13} \mathrm{~cm}$ by requiring that the synchrotron optical photons are self-absorbed.

4. The data can be also interpreted within a two-zone model where $\mathrm{X} / \gamma$-rays are from a near zone while the optical emission is from a far zone. The dissipative photosphere model is inconsistent with the prompt emission data.

5. The broadband afterglow can be interpreted within the standard RS + FS model. Two scenarios are possible: Scenario I invokes both FS and RS to peak at 1100 s, while Scenario II invokes RS only to peak at $1100 \mathrm{~s}$, with the FS peak later when $v_{m}$ cross the optical band. In any case, this is the first time that a rising RS-before its passage of the GRB ejecta (not after, when the RS emission is fast decaying, like in GRB 990123 and a few other cases)—was observed in great detail.

6. In either scenario, the optical peak time can be used to estimate the initial Lorentz factor of GRB ejecta, which is found to be $\Gamma_{0} \approx 250$.

7. From the RS/FS modeling, we infer that the magnetic field strength ratio in reverse and forward shocks is $\Re_{B} \equiv$ $B_{r} / B_{f} \sim 7$. This suggests that the GRB ejecta carry a magnetic flux from the central engine.

8. Jet break modeling reveals that the GRB ejecta are collimated, with an opening angle $\sim 4$. 1 (Scenario I) or $\sim 3.2$ (Scenario II). The jet-corrected $\gamma$-ray energy is $E_{\gamma} \sim$ $1.2 \times 10^{51}$ erg or $E_{\gamma} \sim 7.2 \times 10^{50} \mathrm{erg}$.

We thank the anonymous referee for helpful comments and suggestions to improve the manuscript. This research is supported by the NASA grant NNX08AV63G and the NSF grant PHY-0801007. R.F.S. is supported by an NSERC Discovery grant. A.P.B., A.A.B., N.P.K., M.J.P., and S.R.O. acknowledge the support from the UK Space Agency. B.Z. acknowledges NASA NNX10AD48G and NSF AST-0908362 for support. M.I. acknowledges support from the CRI grant 2009-0063616, funded by MEST of the Korean government. The Centre for Allsky Astrophysics is an Australian Research Council Centre of Excellence, funded by grant CE11E0090. This research made use of public data supplied by the High Energy Astrophysics Science Archive Research Center (HEASARC) at the NASA 
Goddard Space Flight Center. This work has been supported by Spanish Junta de Andalucía through program FQM-02192 and from the Spanish Ministry of Science and Innovation through Projects (including FEDER funds) AYA 2009-14000-C03-01 and AYA2008-03467/ESP. We thank INTA and EELM-CSIC for hosting the BOOTES observatories. The work is based partly on data acquired at the Centro Astronómico Hispano Alemán (CAHA) de Calar Alto and Observatorio de Sierra Nevada (OSN). This research was also supported by the UK STFC.

\section{REFERENCES}

Abdo, A. A., Ackermann, M., Ajello, M., et al. 2009, Nature, 462, 331

Akerlof, C. W., Balsano, R., Barthelmy, S., et al. 1999, Nature, 398, 400

Akerlof, C. W., \& Swan, H. F. 2007, ApJ, 671, 1868

Andreev, M., Sergeev, A., \& Pozanenko, A. 2011, GCN Circ., 11641,

Atwood, W. B., Abdo, A. A., Ackermann, M., et al. 2009, ApJ, 697, 1071

Band, D., Matteson, J., Ford, L., et al. 1993, ApJ, 413, 281

Baltz, E. A., \& Hui, L. 2005, ApJ, 618, 403

Barthelmy, S., Barbier, L. M., Cummings, J. R., et al. 2005, Space Sci. Rev., 120,143

Beardmore, A. P., Baumgartner, W. H., Burrows, D. N., et al. 2011, GCN Circ., 11629,1

Beloborodov, A. M. 2010, MNRAS, 407, 1033

Blandford, R. D., \& McKee, C. F. 1976, Phys. Fluids, 19, 1130

Breeveld, A. A., Curran, P. A., Hoversten, E. A., et al. 2010, MNRAS, 406, 1687

Breeveld, A. A., Landsman, W., Holland, S. T., et al. 2011, in AIP Conf. Proc., 1358, Gamma Ray Bursts 2010 (Melville, NY: AIP), 373

Burrows, D. N., Hill, J. E., Nousek, J. A., et al. 2005a, Space Sci. Rev., 120, 165

Burrows, D. N., Romano, P., Falcone, A., et al. 2005b, Science, 309, 1833

Cenko, S. B., Hora, J., \& Bloom, S. 2011, GCN Circ., 11638, 1

Cenko, S. B., Kasliwal, M., Harrison, F. A., et al. 2006, ApJ, 652, 490

Chester, M. M., \& Beardmore, A. P. 2011, GCN Circ., 11634, 1

Chincarini, G., Moretti, A., Romano, P., et al. 2007, ApJ, 671, 1903

Cucchiara, A., Cenko, S. B., Bloom, J. S., et al. 2011, ApJ, 743, 154

Corsi, A., Piro, L., Kuulkers, E., et al. 2005, A\&A, 438, 829

Covino, S., Lazzati, D., Ghisellini, G., et al. 1999, A\&A, 348, L1

Covino, S., Malesani, D., Ghisellini, G., et al. 2003, A\&A, 400, L9

Dai, Z. G., \& Wu, X. F. 2003, ApJ, 591, L21

Daigne, F., \& Mochkovitch, R. 1998, MNRAS, 296, 275

De Pasquale, M., Piro, L., Perna, R., et al. 2003, ApJ, 592, 1018

De Pasquale, M., Schady, P., Kuin, N. P. M., et al. 2010, ApJ, 709, 146

Efimov, Y., Antoniuk, K., Rumyantsev, V., \& Pozanenko, A. 2003, GCN Circ., 2144, 1

Evans, P. A., Beardmore, A. P., Page, K. L., et al. 2007, A\&A, 469, 379

Evans, P. A., Beardmore, A. P., Page, K. L., et al. 2009, MNRAS, 397, 1177

Evans, P. A., Willingale, R., Osborne, J. P., et al. 2010, A\&A, 519, 102

Fan, Y.-Z., Dai, Z.-G., Huang, Y.-F., \& Lu, T. 2002, Chin. J. Astron. Astrophys, 2, 449

Fan, Y. Z., \& Wei, D. 2005, MNRAS, 364, L42

Fan, Y. Z., Zhang, B., \& Wei, D. M. 2009, Phys. Rev. D, 79, 021301

Fruchter, A., Krolik, J. H., \& Rhoads, J. E. 2001, ApJ, 563, 597

Galama, T. J., Groot, P. J., van Paradijs, J., et al. 1998, ApJ, 497, L13

Gao, W. 2011, Res. Astron. Astrophys., 11, 1317

Garnavich, P. M., Loeb, A., \& Stanek, K. Z. 2000, ApJ, 544, L11

Gaudi, B. S., Granot, J., \& Loeb, A. 2001, ApJ, 561, 178

Gehrels, N., Chincarini, G., Giommi, P., et al. 2004, ApJ, 611, 1005

Gendre, B., Atteia, J. L., Boër, M., et al. 2011, ApJ, 748, 59

Giannios, D. 2008, A\&A, 480, 305

Ghisellini, G., Celotti, A., \& Lazzati, D. 2000, MNRAS, 313, L1

Ghisellini, G., \& Lazzati, D. 1999, ApJ, 309, 7

Golenetskii, S., Aptekar, R., Mazets, E., et al. 2011, GCN Circ., 11659, 1

Gorosabel, J., Duffard, R., Kubanek, P., \& Guijarro, A. 2011, GCN Circ., 11696, 1

Granot, J., \& Sari, R. 2002, ApJ, 568, 820

Greiner, J., Klose, S., Reinsch, K., et al. 2003, Nature, 426, 157

Gruber, D., Krühler, T., Foley, S., et al. 2011, A\&A, 528, 15

Gruzinov, A., \& Waxman, E. 1999, ApJ, 511, 852

Hentunen, V. P., Nissinen, M., \& Salmi, T. 2011, GCN Circ., 11637, 1

Huang, K. Y., Urata, Y., Filippenko, A. V., et al. 2005, ApJ, 628, L93

Im, M., \& Urata, Y. 2011, GCN Circ., 11643, 1

Jacobson, P., \& Fynbo, J. 2010, in AIP Conf. Proc. 1279, Deciphering the Ancient Universe with Gamma-ray Bursts, ed. N. Kawai \& S. Nagataki (Terrsa, Kyoto, Japan: AIP), 167
Jin, Z., \& Fan, Y. 2007, MNRAS, 378, 1043

Jung, G. V. 1989, ApJ, 338, 972

Kalberla, P. M., Burton, W. B., Hartmann, D., et al. 2005, A\&A, 440, 775

Klebesadel, R., Strong, I., \& Olson, R. 1973, ApJ, 182, L85

Klotz, A., Gendre, B., Lass, M., Boer, M., \& Atteia, J. L. 2011a, GCN Circ., 11630,1

Klotz, A., Gendre, B., Lass, M., Boer, M., \& Atteia, J. L. 2011b, GCN Circ., 11632,1

Kobayashi, S. 2000, ApJ, 545, 807

Kobayashi, S., Zhang, B., Mészáros, P., \& Burrows, D. N. 2007, ApJ, 655, 391

Kong, S. W., Wong, A. Y. L., Huang, Y. F., \& Cheng, K. S. 2010, MNRAS, 402, 409

Kumar, P., \& McMahon, R. 2008, MNRAS, 384, 33

Kumar, P., \& Panaitescu, A. 2000, ApJ, 541, L51

Kumar, P., \& Panaitescu, A. 2008, MNRAS, 391, L19

Lazzati, D., \& Begelman, M. C. 2010, ApJ, 725, 1137

Lazzati, D., Covino, S., \& Ghisellini, G. 2002a, MNRAS, 330, 583

Lazzati, D., Morsony, B. J., \& Begelman, M. C. 2009, ApJ, 700, 47

Lazzati, D., Morsony, B. J., \& Begelman, M. C. 2011, ApJ, 732, 34

Lazzati, D., Rossi, E., Covino, S., Ghisellini, G., \& Malesani, D. 2002b, A\&A, 396, L5

Lee, I., Im, M., \& Urata, Y. 2010, J. Korean Astron. Soc., 43, 95

Li, Z., \& Waxman, E. 2008, ApJ, 674, L65

Liang, E., Yi, S., Zhang, J., et al. 2010, ApJ, 725, 2209

Liang, E., Zhang, B., O'Brien, P. T., et al. 2006, ApJ, 646, 351

Mao, J., Malesani, D., D’Avanzo, P., et al. 2012, A\&A, 538, A1

Medvedev, M. V. 2006, ApJ, 637, 869

Medvedev, M. V., \& Loeb, A. 1999, ApJ, 526, 697

Meegan, C., Lichti, G., Bhat, P. N., et al. 2009, ApJ, 702, 791

Mészáros, P. 2006, Rep. Prog. Phys., 69, 2259

Mészáros, P., \& Rees, M. J. 1997, ApJ, 476, 232

Mészáros, P., \& Rees, M. J. 1999, MNRAS, 306, L39

Molinari, E., Vergani, S. D., Malesani, D., et al. 2007, A\&A, 469, 13

Nakar, E., \& Granot, J. 2007, MNRAS, 380, 1744

Nardini, M., Greiner, J., Krühler, T., et al. 2011, A\&A, 531, 39

Nishikawa, K.-I., Niemiec, J., Hardee, P. E., et al. 2009, ApJ, 698, L10

Oates, S. R., Page, M. J., Schady, P., et al. 2009, MNRAS, 395, 490

Ovaldsen, J.-E., Jaunsen, A. O., Fynbo, J. P. U., et al. 2007, ApJ, 662, 294

Pal'shin, V. 2011, GCN Circ., 11697, 1

Panaitescu, A., \& Kumar, P. 2004, MNRAS, 353, 511

Panaitescu, A., \& Vestrand, W. T. 2011, MNRAS, 414, 3537

Pe'er, A., Mészáros, P., \& Rees, M. J. 2006, ApJ, 642, 995

Pe'er, A., \& Waxman, E. 2005, ApJ, 633, 1018

Pe'er, A., \& Zhang, B. 2006, ApJ, 653, 454

Piran, T. 2005, in AIP Conf. Proc. 784, Magnetic Fields in the Universe, (Melville, NY: AIP), 164

Piran, T., Sari, R., \& Zou, Y. C. 2009, MNRAS, 393, 1107

Poole, G., Breeveld, A. A., Page, M. J., et al. 2008, MNRAS, 383, 627

Pozanenko, A. S., Rumyantsev, V. V., Loznikov, V. M., Volnova, A. A., \& Shulga, A. P. 2008, Astron. Lett., 34, 141

Quimby, R. M., Rykoff, E. S., Yost, S. A., et al. 2006, ApJ, 640, 402

Racusin, J. L., Karpov, S. V., Sokolowski, M., et al. 2008, Nature, 455, 183

Rees, M. J., \& Mészáros, P. 1992, MNRAS, 258, 41P

Rees, M. J., \& Mészáros, P. 1994, ApJ, 430, L93

Roming, P. W. A., Kennedy, T. E., Mason, K. O., et al. 2005, Space Sci. Rev., 120,95

Rossi, A., Schulze, S., Klose, S., et al. 2011, A\&A, 529, 142

Rothschild, R. E., Blanco, P. R., Gruber, D. E., et al. 1998, ApJ, 496, 538

Rykoff, E. S., Aharonian, F., Akerlof, C. W., et al. 2009, ApJ, 702, 489

Sahu, D. K., \& Anto, P. 2011, GCN Circ., 11670, 1

Sakamoto, T., Barthelmy, S. D., Baumgartner, W. H., et al. 2011a, ApJS, 195, 2

Sakamoto, T., Barthelmy, S. D., Baumgartner, W. H., et al. 2011b, GCN Circ., 11692,1

Sakamoto, T., Pal'Shin, V., Yamaoka, K., et al. 2011c, PASJ, 63, 215

Sari, R. 1999, ApJ, 524, 43

Sari, R., \& Esin, A. A. 2001, ApJ, 548, 787

Sari, R., \& Piran, T. 1995, ApJ, 455, L143

Sari, R., \& Piran, T. 1999, ApJ, 517, L109

Sari, R., Piran, T., \& Halpern, J. 1999, ApJ, 519, L17

Sari, R., Piran, T., \& Narayan, R. 1998, ApJ, 497, L17

Savaglio, S., Glazebrook, K., \& LeBorgne, D. 2009, ApJ, 691, 182

Schaefer, B. E., Flewelling, H., Rujopakarn, W., \& Guver, T. 2011, GCN Circ., 11631,1

Schlegel, D. J., Finkbeiner, D. P., \& Davis, M. 1998, ApJ, 500, 525

Shen, R., Kumar, P., \& Piran, T. 2010, MNRAS, 403, 229

Shen, R., \& Matzner, C. 2012, ApJ, 744, 36

Shen, R., \& Zhang, B. 2009, MNRAS, 398, 1936 
Silva, R., Fumagalli, M., Worseck, G., \& Prochaska, X. 2011, GCN Circ., 11635, 1

Sizun, P., Shrader, C. R., Attiéé, D., et al. 2004, in Proc. 5th INTEGRAL Workshop on the INTEGRAL Universe, ed. V. Schönfelder, G. Lichti, \& C. Winkler (ESA SP-552; Noordwijk: ESA), 815

Smith, J. A., Tucker, D. L., Kent, S., et al. 2002, AJ, 123, 2121

Sugita, S., Yamaoka, K., Vasquez, N., et al. 2011, GCN Circ., 11682, 1

Tagliaferri, G., Goad, M., Chincarini, G., et al. 2005, Nature, 436, 985

Toma, K., Wu, X.-F., \& Mészáros, P. 2011, MNRAS, 415, 1663

Ukwatta, T., Sonbas, E., Gehrels, N., et al. 2011, GCN Circ., 11655, 1

Updike, A. C., Haislip, J. B., Nysewander, M. C., et al. 2008, ApJ, 685, 361

Urata, Y., Chuang, C., \& Huang, K. 2011, GCN Circ., 11648, 1

van Eerten, H. J., \& MacFadyen, A. I. 2011, ApJ, 733, 37

Volnova, A., Klunko, E., \& Pozanenko, A. 2011, GCN Circ., 11672, 1

Volnova, A., Pozanenko, A., Klunko, E., \& Korobtsev, I. 2010, GCN Circ., 11270,1
Vreeswijk, P., Groot, P., Carter, P., et al. 2011, GCN Circ., 11640, 1

Vurm, I., Beloborodov, A. M., \& Poutanen, J. 2011, ApJ, 738, 77

Waxman, E., \& Draine, B. T. 2000, ApJ, 537, 796

Weibel, E. S. 1959, Phys. Rev. Lett., 2, 83

Wijers, R., Rees, M., \& Mészáros, P. 1997, MNRAS, 288, 51

Xue, R., Fan, Y., \& Wei, D. 2009, A\&A, 498, 671

Yamaoka, K., Endo, A., Enoto, T., et al. 2009, PASJ, 61, 35

Yu, Y. W., Wang, X. Y., \& Dai, Z. G. 2009, ApJ, 692, 1662

Zhang, B. 2011, C. R. Phys., 12, 206

Zhang, B., Fan, Y. Z., Dyks, J., et al. 2006, ApJ, 642, 354

Zhang, B., Kobayashi, S., \& Meśzaŕos, P. 2003, ApJ, 595, 950

Zhang, B., \& Mészáros, P. 2002, ApJ, 581, 1236

Zhang, B., \& Mészáros, P. 2004, Int. J. Mod. Phys. A, 19, 2385

Zhang, B., \& Yan, H. 2011, ApJ, 726, 90

Zou, Y., Wu, X., \& Dai, Z. 2005, MNRAS, 363, 93 